\title{
High-Order Optimal Edge Elements for Pyramids, Prisms and Hexahedra
}

\author{
Morgane Bergot ${ }^{\mathrm{a}}$, Marc Duruflé ${ }^{\mathrm{b}}$ \\ ${ }^{a}$ CALVI project team, INRIA Nancy-Grand Est, Strasbourg, FRANCE \\ morgane.bergot@inria.fr \\ ${ }^{b}$ BACCHUS project team, INRIA Bordeaux Sud-Ouest, Bordeaux, FRANCE \\ marc.durufle@inria.fr
}

\begin{abstract}
Edge elements are a popular method to solve Maxwell's equations especially in time-harmonic domain. However, when non-affine elements are considered, elements of the Nédélec's first family (Nédélec, 1980) are not providing an optimal rate of the convergence of the numerical solution toward the solution of the exact problem in $H(\mathrm{curl})$ norm. We propose new finite element spaces for pyramids, prisms, and hexahedra in order to recover the optimal convergence. In the particular case of pyramids, a comparison with other existing elements found in the literature is performed. Numerical results show the good behaviour of these new finite elements.
\end{abstract}

Keywords: Edge Elements, High-order finite element, Pyramids, Maxwell's equations

\section{Introduction}

We are interested in the resolution of 3-D time-harmonic Maxwell's equations

$$
-\omega^{2} \varepsilon E+\operatorname{curl}\left(\frac{1}{\mu} \operatorname{curl} E\right)=f
$$

where $\varepsilon, \mu$ may be heterogeneous. Many numerical methods have been developed to solve this equation. Using nodal finite elements is possible by considering either a weighted regularization (Costabel and Dauge, 2002) or a Discontinuous Galerkin formulation (Houston et al., 2005). A more natural choice consists of using edge elements, and considering the following variational formulation:

$$
-\omega^{2} \int_{\Omega} \varepsilon E \cdot \varphi d x+\int_{\Omega} \frac{1}{\mu} \operatorname{curl} E \cdot \operatorname{curl} \varphi d x=\int_{\Omega} f \cdot \varphi d x
$$

We restrict ourselves to the study of Nédélec's first family elements since optimal convergence of the convergence of the numerical solution toward the solution of the exact problem is sought in $H($ curl $)$-norm. These elements are well-known in the case of tetrahedra, prisms, and hexahedra (see for example (Monk, 2002)). Concerning pyramids, the litterature is detailed in the paper.

When we consider a family of meshes relatively well structured, the elements are usually tending to affine elements. This property can be characterized since the jacobian matrix of these elements is tending to a constant matrix. It has been shown by Demkowicz and coworkers ((Demkowicz et al., 2007)) that for this type of meshes, Nédélec's first family provide on optimal rate of convergence Here, we consider a family of meshes for which the jacobian matrix does not tend to a constant matrix, which is the case for general unstructured meshes. In this case, these elements are not providing an optimal convergence $O\left(h^{r}\right)$ in $H(c u r l)$-norm when non-affine hexahedra and prisms are considered, that is to say when the transformation from the reference element to the real element of the mesh is in $\mathbb{Q}_{1}$ instead of being affine.

An improved finite element space has been proposed in (Arnold et al., 2005) in 2-D for quadrilaterals, and in 3-D in (Falk et al., 2011) for first-order hexahedra. We propose here optimal finite element spaces in 3-D for hexahedra, prisms and pyramids at any order of approximation. Nodal basis functions are constructed by inverting a Vandermonde matrix, but this matrix appears to be ill-conditioned for pyramids. Hierarchical basis functions are given for all the 
types of elements, with a tensor structure, which can be exploited to obtain a fast matrix-vector product or a fast computation of finite element matrix.

In the special case of pyramidal elements, we propose a finite element space compatible with spaces of Nédélec's first family of other elements. Moreover, a comparison is performed with pyramidal edge elements proposed in the literature, e.g. (Coulomb et al., 1997), (Nigam and Phillips, 2010a), (Graglia et al., 1999).

\section{Optimal Finite Element Spaces}

Let us introduce the following classical spaces:

Definition 1. $\quad$ In 2-D:

$$
\begin{gathered}
\mathbb{Q}_{m, n}(x, y)=\operatorname{Span}\left\{x^{i} y^{j}, 0 \leq i \leq m, 0 \leq j \leq n\right\} \\
\mathbb{P}_{r}(x, y)=\operatorname{Span}\left\{x^{i} y^{j}, i, j \geq 0, i+j \leq r\right\} \\
\widetilde{\mathbb{P}}_{r}(x, y)=\operatorname{Span}\left\{x^{i} y^{j}, i, j \geq 0, i+j=r\right\} \\
\mathcal{S}_{r}(x, y)=\left\{u=\left(u_{1}, u_{2}\right) \in \widetilde{\mathbb{P}}_{r}^{2}, \text { such that } u_{1} x+u_{2} y=0\right\} \\
\mathcal{R}_{r}(x, y)=\left(\mathbb{P}_{r-1}(x, y)\right)^{2} \oplus \mathcal{S}_{r}(x, y)
\end{gathered}
$$

- In 3-D:

$$
\begin{gathered}
\mathbb{Q}_{m, n, p}(x, y, z)=\operatorname{Span}\left\{x^{i} y^{j} z^{k}, 0 \leq i \leq m, 0 \leq j \leq n, 0 \leq k \leq p\right\} \\
\mathbb{P}_{r}(x, y, z)=\operatorname{Span}\left\{x^{i} y^{j} z^{k}, i, j, k \geq 0, i+j+k \leq r\right\} \\
\widetilde{\mathbb{P}}_{r}(x, y, z)=\operatorname{Span}\left\{x^{i} y^{j} z^{k}, i, j, k \geq 0, i+j+k=r\right\} \\
\mathcal{S}_{r}(x, y, z)=\left\{u \in \widetilde{\mathbb{P}}_{r}^{3} \text { so that } u_{1} x+u_{2} y+u_{3} z=0\right\} \\
\mathcal{R}_{r}(x, y, z)=\mathbb{P}_{r-1}^{3} \oplus \mathcal{S}_{r}
\end{gathered}
$$

$\mathcal{R}_{r}$ is the Nédélec's first family introduced by Nédélec (Nédélec, 1980). We also introduce the approximation space for continuous pyramidal element (see (Bergot et al., 2010))

$$
\mathbb{B}_{r}=\mathbb{P}_{r}(x, y, z) \oplus \sum_{k=0}^{r-1} \mathbb{P}_{k}(x, y)\left(\frac{x y}{1-z}\right)^{r-k}
$$

and this space written on the cube $[-1,1] \times[0,1]$ :

$$
\mathbb{C}_{r}=\operatorname{Span}\left\{x^{i} y^{j}(1-z)^{k}, 0 \leq i, j \leq k \leq r\right\}
$$

The finite element approximation is constructed by considering a transformation $F$ from a reference element $\hat{K}$ (unit tetrahedron, symmetric pyramid, unit prism, unit cube) to a real element $K$ on the mesh. This transformation is written as

$$
F=\sum_{1 \leq i \leq n_{i}} S_{i} \hat{\varphi}_{i}^{1}
$$

where $n_{i}$ is the number of vertices of the element, $S_{i}=\left(x_{i}, y_{i}, z_{i}\right)$ are the vertices and $\hat{\varphi}_{i}^{1}$ are mapping functions depending on the considered type of the element. 


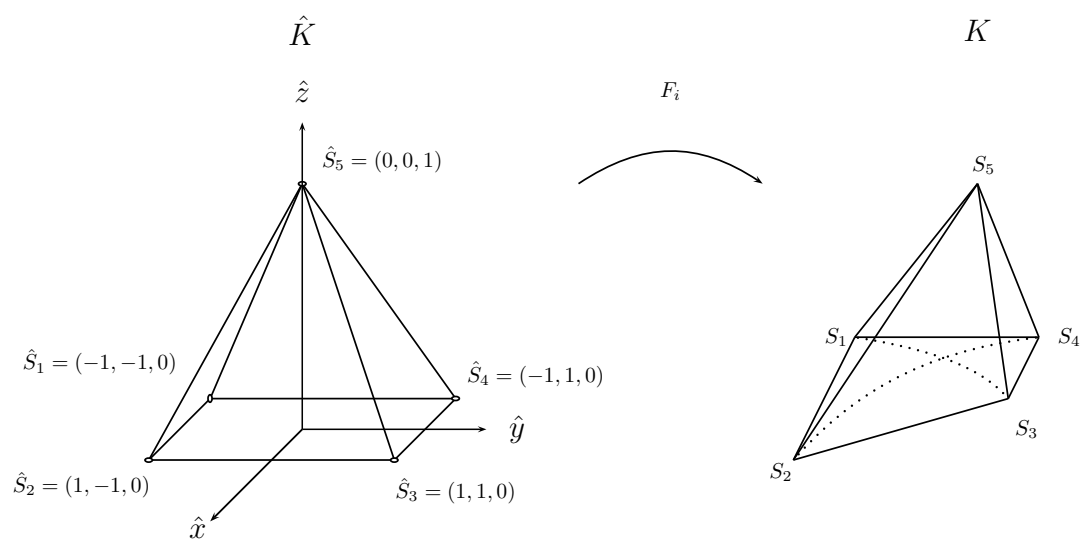

Figure 1: transformation $\mathrm{F}$ for a pyramid

For pyramidal elements, the mapping functions given in (Bedrosian, 1992) using rational fractions are the following:

$$
\left\{\begin{array}{l}
\hat{\varphi}_{1}^{1}=\frac{1}{4}\left(1-\hat{x}-\hat{y}-\hat{z}+\frac{\hat{x} \hat{y}}{1-\hat{z}}\right) \\
\hat{\varphi}_{2}^{1}=\frac{1}{4}\left(1+\hat{x}-\hat{y}-\hat{z}-\frac{\hat{x} \hat{y}}{1-\hat{z}}\right) \\
\hat{\varphi}_{3}^{1}=\frac{1}{4}\left(1+\hat{x}+\hat{y}-\hat{z}+\frac{\hat{x} \hat{y}}{1-\hat{z}}\right) \\
\hat{\varphi}_{4}^{1}=\frac{1}{4}\left(1-\hat{x}+\hat{y}-\hat{z}-\frac{\hat{x} \hat{y}}{1-\hat{z}}\right) \\
\hat{\varphi}_{5}^{1}=\hat{z}
\end{array}\right.
$$

The obtained transformation is shown in Fig. 1

For the other types of elements, the transformation is classical (see (Šolín et al., 2003)).

Let us denote

$$
H(\operatorname{curl}, \Omega)=\left\{u \in\left(L^{2}(\Omega)\right)^{3} \text { such that } \operatorname{curl} u \in\left(L^{2}(\Omega)\right)^{3}\right\}
$$

The electric field $E$ and test function $\varphi$ of the variational formulation (2) are belonging to the following finite element space:

$$
V_{h}=\left\{u \in H(\operatorname{curl}, \Omega) \text { so that }\left.u\right|_{K} \in P_{r}^{F}\right\}
$$

where $P_{r}^{F}(K)$ denotes the finite element space on the real element $K$. This space is induced by the choice of the space $\hat{P}_{r}(\hat{K})$ thanks to Piola $H(c u r l)$-conforming transform

$$
P_{r}^{F}(K)=\left\{u \text { so that } D F^{*} u \circ F \in \hat{P}_{r}(\hat{K})\right\}
$$

The space $\hat{P}_{r}(\hat{K})$ is independent of the element $K$, since it depends only on the reference element $\hat{K}$ and on the order of approximation $r$. We denote by $D F$ the jacobian matrix of transformation $F$.

In order to obtain an optimal convergence in $O\left(h^{r}\right)$ for $H(c u r l)$-norm, where $h$ denotes the mesh size, a sufficient condition (see (Monk, 2002)) is that the space $P_{r}^{F}$ includes all polynomials of space $\mathcal{R}_{r}$.

Definition 2. $\hat{P}_{r}(\hat{K})$ is said to be optimal if for any element $K, P_{r}^{F} \supset \mathcal{R}_{r}$

After a careful examination of $D F^{*} \mathbf{p} \circ F$ for all the polynomials $\mathbf{p}$ in $\mathcal{R}_{r}$, the following optimal finite element spaces can be obtained. 
Theorem 1. The optimal space $\hat{P}_{r}(\hat{K})$ is equal to:

- Tetrahedron:

$$
\hat{P}_{r}(\hat{K})=\mathcal{R}_{r}
$$

- Hexahedron:

$$
\hat{P}_{r}(\hat{K})=\mathcal{Q}_{r}=\mathbb{Q}_{r-1, r+1, r+1} \times \mathbb{Q}_{r+1, r-1, r+1} \times \mathbb{Q}_{r+1, r+1, r-1}
$$

- Prism:

$$
\hat{P}_{r}(\hat{K})=\mathcal{W}_{r}=\left(\mathcal{R}_{r}(\hat{x}, \hat{y}) \otimes \mathbb{P}_{r+1}(\hat{z})\right) \times\left(\mathbb{P}_{r+1}(\hat{x}, \hat{y}) \otimes \mathbb{P}_{r-1}(\hat{z})\right)
$$

- Pyramid:

$$
\begin{aligned}
& \hat{P}_{r}(\hat{K})=\mathcal{B}_{r}=\mathbb{B}_{r-1}^{3} \oplus\left\{\frac{\hat{x}^{p} \hat{y}^{p}}{(1-\hat{z})^{p+2}}\left[\begin{array}{c}
\hat{y}(1-\hat{z}) \\
\hat{x}(1-\hat{z}) \\
\hat{x} \hat{y}
\end{array}\right], 0 \leq p \leq r-1\right\} \\
& \oplus\left\{\frac{\hat{x}^{m} \hat{y}^{n+2}}{(1-\hat{z})^{m+2}}\left[\begin{array}{c}
(1-\hat{z}) \\
0 \\
\hat{x}
\end{array}\right], \frac{\hat{x}^{n+2} \hat{y}^{m}}{(1-\hat{z})^{m+2}}\left[\begin{array}{c}
0 \\
(1-\hat{z}) \\
\hat{y}
\end{array}\right], 0 \leq m \leq n \leq r-2 l\right\} \\
& \oplus\left\{\frac{\hat{x}^{p} \hat{y}^{q}}{(1-\hat{z})^{p+q+1-r}}\left[\begin{array}{c}
(1-\hat{z}) \\
0 \\
\hat{x}
\end{array}\right], \frac{\hat{x}^{q} \hat{y}^{p}}{(1-\hat{z})^{p+q+1-r}}\left[\begin{array}{c}
0 \\
(1-\hat{z}) \\
\hat{y}
\end{array}\right], \begin{array}{l}
0 \leq p \leq r-1 \\
0 \leq q \leq r+1
\end{array}\right\}
\end{aligned}
$$

If we denote $T$ the transformation from the cube $[-1,1]^{2} \times[0,1]$ to the symmetric pyramid:

$$
\begin{aligned}
& \hat{x}=(1-\tilde{z}) \tilde{x} \\
& \hat{y}=(1-\tilde{z}) \tilde{y} \\
& \hat{z}=\tilde{z}
\end{aligned}
$$

The optimal space of the pyramid on the cube is equal to

$$
\begin{aligned}
\mathcal{C}_{r}= & \mathcal{B}_{r}(\hat{x}, \hat{y}, \hat{z}) \circ T \\
= & \left.\left(\mathbb{B}_{r-1} \circ T(\tilde{x}, \tilde{y}, \tilde{z})\right)^{3} \oplus\left\{\tilde{x}^{p} \tilde{y}^{p}(1-\tilde{z})^{p}\left[\begin{array}{c}
\tilde{y} \\
\tilde{x} \\
\tilde{x} \tilde{y}
\end{array}\right], \quad 0 \leq p \leq r-1\right\}\right\} \\
& \oplus\left\{\tilde{x}^{m} \tilde{y}^{n+2}(1-\tilde{z})^{n+1}\left[\begin{array}{l}
1 \\
0 \\
\tilde{x}
\end{array}\right], \tilde{x}^{n+2} \tilde{y}^{m}(1-\tilde{z})^{n+1}\left[\begin{array}{l}
0 \\
1 \\
\tilde{y}
\end{array}\right], \quad 0 \leq m \leq n \leq r-2\right\} \\
& \left.\oplus\left\{\begin{array}{l}
1 \\
0 \\
\tilde{x}
\end{array}\right], \tilde{x}^{q} \tilde{y}^{q}(1-\tilde{z})^{r}(1-\tilde{z})^{r}\left[\begin{array}{l}
0 \\
1 \\
\tilde{y}
\end{array}\right], \begin{array}{l}
0 \leq p \leq r-1 \\
0 \leq q \leq r+1
\end{array}\right\}
\end{aligned}
$$

One should remark that we have applied a simple change of variables on the cube, and not an H(curl)-conforming transformation since $D T^{*}$ has not been applied. 
Proof. The proof is done only for pyramids since this case is the most interesting and difficult to treat. Similar computations have been done for hexahedra and triangular prisms but are not reported here. The proof is performed by considering a monomial $\mathbf{p}(x, y, z)$ in $\mathcal{R}_{r}$, we compute $\hat{p}=\mathbf{p} \circ F$, and Piola transform $D F^{*}$ is applied to $\hat{p}$ and we search all the monomials in $(\hat{x}, \hat{y}, \hat{z})$ independent of $F$ that are combined to obtain $D F^{*} \mathbf{p} \circ F$. The transformation $F$ can be written in the form:

$$
F(\hat{x}, \hat{y}, \hat{z})=A_{0}+A_{1} \hat{x}+A_{2} \hat{y}+A_{3} \hat{z}+\frac{\hat{x} \hat{y}}{1-\hat{z}} C
$$

where $A_{1}, A_{2}, A_{3}$ and $C$ belong to $\mathbb{R}^{3}$ and depend on the geometry. The transformation $F$ is polynomial in $(\tilde{x}, \tilde{y}, \tilde{z})$ (variables in the cube):

$$
F(\tilde{x}, \tilde{y}, \tilde{z})=A_{0}+A_{1} \tilde{x}(1-\tilde{z})+A_{2} \tilde{y}(1-\tilde{z})+A_{3} \tilde{z}+\tilde{x} \tilde{y}(1-\tilde{z}) C
$$

The derivatives of $F$ are equal to:

$$
\begin{gathered}
\frac{\partial F}{\partial \hat{x}}=A_{1}+\frac{\hat{y}}{1-\hat{z}} C=A_{1}+C \tilde{y} \\
\frac{\partial F}{\partial \hat{y}}=A_{2}+\frac{\hat{x}}{1-\hat{z}} C=A_{2}+C \tilde{x} \\
\frac{\partial F}{\partial \hat{z}}=A_{3}+\frac{\hat{x} \hat{y}}{(1-\hat{z})^{2}} C=A_{3}+C \tilde{x} \tilde{y}
\end{gathered}
$$

For a polynomial $p$ in $\mathcal{R}_{r}, \hat{p}=\mathbf{p} \circ F$ is the expression of $\mathbf{p}$ in the symmetric pyramid $\hat{K}$ and $\tilde{p}$ the expression on the cube $[-1,1]^{2} \times[0,1]$. When Piola transform $D F^{*}$ is applied to $\hat{p}$ :

$$
D F^{*} \hat{p}=\left[\begin{array}{c}
\left(A_{1}+C \tilde{y}\right) \cdot \tilde{p} \\
\left(A_{2}+C \tilde{x}\right) \cdot \tilde{p} \\
\left(A_{3}+C \tilde{x} \tilde{y}\right) \cdot \tilde{p}
\end{array}\right]
$$

For $\mathbf{p} \in \mathbb{P}_{r-1}^{3}$, as it has been proven in (Bergot et al., 2010), one can equivalently consider $\tilde{p} \in \mathbb{C}_{r-1}^{3}$ :

$$
\tilde{p}=\tilde{x}^{i} \tilde{y}^{j}(1-\tilde{z})^{k} E_{0}
$$

where $i, j \leq k \leq r-1$ and $E_{0}$ a constant vector of $\mathbb{R}^{3}$. One gets:

$$
D F^{*} \tilde{p}=\left[\begin{array}{c}
A_{1} \cdot E_{0} \tilde{x}^{i} \tilde{y}^{j}(1-\tilde{z})^{k}+C \cdot E_{0} \tilde{x}^{i} \tilde{y}^{j+1}(1-\tilde{z})^{k} \\
A_{2} \cdot E_{0} \tilde{x}^{i} \tilde{y}^{j}(1-\tilde{z})^{k}+C \cdot E_{0} \tilde{x}^{i+1} \tilde{y}^{j}(1-\tilde{z})^{k} \\
A_{3} \cdot E_{0} \tilde{x}^{i} \tilde{y}^{j}(1-\tilde{z})^{k}+C \cdot E_{0} \tilde{x}^{i+1} \tilde{y}^{j+1}(1-\tilde{z})^{k}
\end{array}\right]
$$

The left side is equal to (when $C=0$, i.e. affine pyramids)

$$
\left[\begin{array}{c}
A_{1} \cdot E_{0} \tilde{x}^{i} \tilde{y}^{j}(1-\tilde{z})^{k} \\
A_{2} \cdot E_{0} \tilde{x}^{i} \tilde{y}^{j}(1-\tilde{z})^{k} \\
A_{3} \cdot E_{0} \tilde{x}^{i} \tilde{y}^{j}(1-\tilde{z})^{k}
\end{array}\right]
$$

belongs to $\mathbb{C}_{r-1}^{3}$. It means that all functions of $\mathbb{C}_{r-1}^{3}$ are needed. For the right side, four cases appear

- $i=j=k=p-1$, with $1 \leq p \leq r$, we then have:

$$
\left[\begin{array}{c}
\tilde{x}^{p-1} \tilde{y}^{p}(1-\tilde{z})^{p-1} \\
\tilde{x}^{p} \tilde{y}^{p-1}(1-\tilde{z})^{p-1} \\
\tilde{x}^{p} \tilde{y}^{p}(1-\tilde{z})^{p-1}
\end{array}\right] \in \mathcal{B}_{r}
$$


- $i=k=m+1$ and $j<k$, then $j \leq m$ and $m \leq r-2$. We have:

$$
\left[\begin{array}{c}
0 \\
\tilde{x}^{m+2} \tilde{y}^{j}(1-\tilde{z})^{m+1} \\
\tilde{x}^{m+2} \tilde{y}^{j+1}(1-\tilde{z})^{m+1} \\
\in \mathcal{B}_{r}
\end{array}\right]+\left[\begin{array}{c}
\tilde{x}^{m+1} \tilde{y}^{j+1}(1-\tilde{z})^{m+1} \\
0 \\
0 \\
\in \mathbb{C}_{r-1}^{3}
\end{array}\right]
$$

- $j=k=m+1$ and $i=p<k$, then $p \leq m$ and $m \leq r-2$. We have:

$$
\left[\begin{array}{c}
\tilde{x}^{p} \tilde{y}^{m+2}(1-\tilde{z})^{m+1} \\
0 \\
\tilde{x}^{p+1} \tilde{y}^{m+2}(1-\tilde{z})^{m+1} \\
\in \mathcal{B}_{r}
\end{array}\right]+\left[\begin{array}{c}
0 \\
\tilde{x}^{p+1} \tilde{y}^{m+1}(1-\tilde{z})^{m+1} \\
0 \\
\in \mathbb{C}^{3}
\end{array}\right]
$$

- $i, j<k$, in this case $D F^{*} \tilde{p} \in \mathbb{C}_{r-1}^{3}$

It can be noticed that in order to generate $\mathbb{P}_{r-1}^{3}$, a necessary and sufficient condition is that $\hat{P}_{r}^{K}$ includes the polynomial space:

$$
\mathbb{C}_{r-1}^{3} \oplus\left[\begin{array}{c}
\tilde{x}^{j} \tilde{y}^{k+2}(1-\tilde{z})^{k+1} \\
0 \\
\tilde{x}^{j+1} \tilde{y}^{k+2}(1-\tilde{z})^{k+1}
\end{array}\right] \oplus\left[\begin{array}{c}
0 \\
\tilde{x}^{k+2} \tilde{y}^{j}(1-\tilde{z})^{k+1} \\
\tilde{x}^{k+2} \tilde{y}^{j+1}(1-\tilde{z})^{k+1}
\end{array}\right] \oplus\left[\begin{array}{c}
\tilde{x}^{p-1} \tilde{y}^{p}(1-\tilde{z})^{p-1} \\
\tilde{x}^{p} \tilde{y}^{p-1}(1-\tilde{z})^{p-1} \\
\tilde{x}^{p} \tilde{y}^{p}(1-\tilde{z})^{p-1}
\end{array}\right]
$$

with $j \leq k \leq r-2,1 \leq p \leq r$. This space should be considered for the construction of edge elements of Nédélec's second family ( $\mathbb{P}_{r}^{3}$ for tetrahedra).

We now consider a function of $\mathcal{S}_{r}$ :

$$
\left[\begin{array}{c}
0 \\
x^{i} y^{j} z^{k+1} \\
-x^{i} y^{j+1} z^{k}
\end{array}\right], i+j \leq r-1, k=r-1-i-j
$$

The degree of $x^{i} y^{j} z^{k}$ is exactly $r-1$, therefore this monomial can be written as a linear combination of $\tilde{x}^{i} \tilde{y}^{j}(1-\tilde{z})^{r-1}$ with $i, j \leq r-1$. In order to simplify the computations, it is equivalent to consider a function of $\mathcal{S}_{r}$ under the form:

$$
\tilde{x}^{i} \tilde{y}^{j}(1-\tilde{z})^{r-1}\left[\begin{array}{l}
0 \\
z \\
-y
\end{array}\right], \quad i, j \leq r-1
$$

Let us denote

$$
E=\left[\begin{array}{c}
0 \\
z \\
-y
\end{array}\right]=\left[\begin{array}{l}
0 \\
\left(A_{0}^{z}-A_{3}^{z}\right)+\tilde{x}(1-\tilde{z}) A_{1}^{z}+\tilde{y}(1-\tilde{z}) A_{2}^{z}-(1-\tilde{z}) A_{3}^{z}+\tilde{x} \tilde{y}(1-\tilde{z}) C^{z} \\
-\left[\left(A_{0}^{y}-A_{3}^{y}\right)+\tilde{x}(1-\tilde{z}) A_{1}^{y}+\tilde{y}(1-\tilde{z}) A_{2}^{y}-(1-\tilde{z}) A_{3}^{y}+\tilde{x} \tilde{y}(1-\tilde{z}) C^{y}\right]
\end{array}\right]
$$

with

$$
\begin{array}{ll}
A_{0}=\left(A_{0}^{x}, A_{0}^{y}, A_{0}^{z}\right), & A_{1}=\left(A_{1}^{x}, A_{1}^{y}, A_{1}^{z}\right), \quad A_{2}=\left(A_{2}^{x}, A_{2}^{y}, A_{2}^{z}\right) \\
A_{3}=\left(A_{3}^{x}, A_{3}^{y}, A_{3}^{z}\right), & C=\left(C^{x}, C^{y}, C^{z}\right)
\end{array}
$$

The first component of $D F^{*} E$ is:

$$
\begin{aligned}
\left(D F^{*} E\right)_{x}= & {\left[A_{1}^{y}\left(A_{0}^{z}-A_{3}^{z}\right)-A_{1}^{z}\left(A_{0}^{y}-A_{3}^{y}\right)\right]+\left[C^{y}\left(A_{0}^{z}-A_{3}^{z}\right)-C^{z}\left(A_{0}^{y}-A_{3}^{y}\right)\right] \tilde{y}+} \\
& {\left[A_{1}^{y} A_{2}^{z}-A_{1}^{z} A_{2}^{y}\right] \tilde{y}(1-\tilde{z})+\left[C^{y} A_{2}^{z}-C^{z} A_{2}^{y}\right] \tilde{y}^{2}(1-\tilde{z}) } \\
& +\left[A_{1}^{z} A_{3}^{y}-A_{1}^{y} A_{3}^{z}\right](1-\tilde{z})+\left[A_{3}^{y} C^{z}-A_{3}^{z} C^{y}\right] \tilde{y}(1-\tilde{z}) \\
= & b_{0}+b_{1} \tilde{y}+\left(b_{2}+b_{3}\right) \tilde{y}(1-\tilde{z})+b_{4}(1-\tilde{z})+b_{5} \tilde{y}^{2}(1-\tilde{z})
\end{aligned}
$$


where

$$
\begin{gathered}
b_{0}=A_{1}^{y}\left(A_{0}^{z}-A_{3}^{z}\right)-A_{1}^{z}\left(A_{0}^{y}-A_{3}^{y}\right) \\
b_{1}=C^{y}\left(A_{0}^{z}-A_{3}^{z}\right)-C^{z}\left(A_{0}^{y}-A_{3}^{y}\right) \\
b_{2}=A_{1}^{y} A_{2}^{z}-A_{1}^{z} A_{2}^{y} \\
b_{3}=A_{3}^{y} C^{z}-A_{3}^{z} C^{y} \\
b_{4}=A_{1}^{z} A_{3}^{y}-A_{1}^{y} A_{3}^{z} \\
b_{5}=C^{y} A_{2}^{z}-C^{z} A_{2}^{y}
\end{gathered}
$$

The second component $D F^{*} E$ reads

$$
\begin{aligned}
\left(D F^{*} E\right)_{y}= & {\left[A_{2}^{y}\left(A_{0}^{z}-A_{3}^{z}\right)-A_{2}^{z}\left(A_{0}^{y}-A_{3}^{y}\right)\right]+\left[C^{y}\left(A_{0}^{z}-A_{3}^{z}\right)-C^{z}\left(A_{0}^{y}-A_{3}^{y}\right)\right] \tilde{x}+} \\
& {\left[A_{2}^{y} A_{1}^{z}-A_{2}^{z} A_{1}^{y}\right] \tilde{x}(1-\tilde{z})+\left[C^{y} A_{1}^{z}-C^{z} A_{1}^{y}\right] \tilde{x}^{2}(1-\tilde{z}) } \\
& +\left[A_{3}^{y} A_{2}^{z}-A_{3}^{z} A_{2}^{y}\right](1-\tilde{z})+\left[A_{3}^{y} C^{z}-A_{3}^{z} C^{y}\right] \tilde{x}(1-\tilde{z}) \\
= & b_{6}+b_{1} \tilde{x}+\left(b_{3}-b_{2}\right) \tilde{x}(1-\tilde{z})+b_{8}(1-\tilde{z})+b_{7} \tilde{x}^{2}(1-\tilde{z})
\end{aligned}
$$

where

$$
\begin{gathered}
b_{6}=A_{2}^{y}\left(A_{0}^{z}-A_{3}^{z}\right)-A_{2}^{z}\left(A_{0}^{y}-A_{3}^{y}\right) \\
b_{7}=C^{y} A_{1}^{z}-C^{z} A_{1}^{y} \\
b_{8}=A_{3}^{y} A_{2}^{z}-A_{3}^{z} A_{2}^{y}
\end{gathered}
$$

The last component is equal to

$$
\begin{aligned}
\left(D F^{*} E\right)_{z}= & {\left[A_{3}^{y} A_{0}^{z}-A_{3}^{z} A_{0}^{y}\right]+\left[C^{y}\left(A_{0}^{z}-A_{3}^{z}\right)-C^{z}\left(A_{0}^{y}-A_{3}^{y}\right)\right] \tilde{x} \tilde{y} } \\
& +\left[A_{1}^{z} A_{3}^{y}-A_{1}^{y} A_{3}^{z}\right] \tilde{x}(1-\tilde{z})+\left[C^{y} A_{1}^{z}-C^{z} A_{1}^{y}\right] \tilde{x}^{2} \tilde{y}(1-\tilde{z})+\left[A_{3}^{y} A_{2}^{z}-A_{3}^{z} A_{2}^{y}\right] \tilde{y}(1-\tilde{z}) \\
& +\left[C^{y} A_{2}^{z}-C^{z} A_{2}^{y}\right] \tilde{x} \tilde{y}^{2}(1-\tilde{z})+2\left[A_{3}^{y} C^{z}-A_{3}^{z} C^{y}\right] \tilde{x} \tilde{y}(1-\tilde{z}) \\
= & b_{9}+b_{1} \tilde{x} \tilde{y}+b_{4} \tilde{x}(1-\tilde{z})+b_{7} \tilde{x}^{2}(1-\tilde{z})+b_{5} \tilde{x} \tilde{y}^{2}(1-\tilde{z}) \\
& +\left(b_{3}-b_{2}\right) \tilde{x} \tilde{y}(1-\tilde{z})+\left(b_{3}+b_{2}\right) \tilde{x} \tilde{y}(1-\tilde{z})+b_{8} \tilde{y}(1-\tilde{z})
\end{aligned}
$$

where

$$
b_{9}=A_{3}^{y} A_{0}^{z}-A_{3}^{z} A_{0}^{y}
$$

We have:

$$
\begin{aligned}
D F^{*} E= & {\left[\begin{array}{c}
b_{0} \\
b_{6} \\
b_{9}
\end{array}\right]+b_{1}\left[\begin{array}{c}
\tilde{y} \\
\tilde{x} \\
\tilde{x} \tilde{y}
\end{array}\right]+\left(b_{2}+b_{3}\right)\left[\begin{array}{c}
\tilde{y}(1-\tilde{z}) \\
0 \\
\tilde{x} \tilde{y}(1-\tilde{z})
\end{array}\right]+\left(b_{3}-b_{2}\right)\left[\begin{array}{c}
0 \\
\tilde{x}(1-\tilde{z}) \\
\tilde{x} \tilde{y}(1-\tilde{z})
\end{array}\right] } \\
& +b_{4}\left[\begin{array}{c}
1-\tilde{z} \\
0 \\
\tilde{x}(1-\tilde{z})
\end{array}\right]+b_{5}\left[\begin{array}{c}
\tilde{y}^{2}(1-\tilde{z}) \\
0 \\
\tilde{x} \tilde{y}^{2}(1-\tilde{z})
\end{array}\right]+b_{7}\left[\begin{array}{c}
0 \\
\tilde{x}^{2}(1-\tilde{z}) \\
\tilde{x}^{2} \tilde{y}(1-\tilde{z})
\end{array}\right]+b_{8}\left[\begin{array}{c}
0 \\
(1-\tilde{z}) \\
\tilde{y}(1-\tilde{z})
\end{array}\right]
\end{aligned}
$$

The function

$$
\tilde{x}^{i} \tilde{y}^{j}(1-\tilde{z})^{r-1}\left[\begin{array}{l}
b_{0} \\
b_{6} \\
b_{9}
\end{array}\right]
$$


belongs to $C_{r-1}^{3}$. The coefficients $b_{1},\left(b_{2}+b_{3}\right),\left(b_{3}-b_{2}\right), b_{4}, b_{5}, b_{7}$ and $b_{8}$, viewed as functions as $A_{0}, A_{1}, A_{2}, A_{3}, C$, are linearly independent, The following functions are necessary and sufficient:

$$
\begin{gathered}
\tilde{x}^{i} \tilde{y}^{j}(1-\tilde{z})^{r-1}\left\{\left[\begin{array}{c}
\tilde{y} \\
\tilde{x} \\
\tilde{x} \tilde{y}
\end{array}\right],\left[\begin{array}{c}
\tilde{y}(1-\tilde{z}) \\
0 \\
\tilde{x} \tilde{y}(1-\tilde{z})
\end{array}\right],\left[\begin{array}{c}
1-\tilde{z} \\
0 \\
\tilde{x}(1-\tilde{z})
\end{array}\right],\left[\begin{array}{c}
\tilde{y}^{2}(1-\tilde{z}) \\
0 \\
\tilde{x} \tilde{y}^{2}(1-\tilde{z})
\end{array}\right]\right\} \\
\tilde{x}^{i} \tilde{y}^{j}(1-\tilde{z})^{r-1}\left\{\left[\begin{array}{c}
0 \\
\tilde{x}(1-\tilde{z}) \\
\tilde{x} \tilde{y}(1-\tilde{z})
\end{array}\right],\left[\begin{array}{c}
0 \\
\tilde{x}^{2}(1-\tilde{z}) \\
\tilde{x}^{2} \tilde{y}(1-\tilde{z})
\end{array}\right],\left[\begin{array}{c}
0 \\
(1-\tilde{z}) \\
\tilde{y}(1-\tilde{z})
\end{array}\right]\right\}
\end{gathered}
$$

We can regroup them in three families:

- A first group

$$
\left[\begin{array}{c}
\tilde{x}^{i} \tilde{y}^{j+1}(1-\tilde{z})^{r-1} \\
\tilde{x}^{i+1} \tilde{y}^{j}(1-\tilde{z})^{r-1} \\
\tilde{x}^{i+1} \tilde{y}^{j+1}(1-\tilde{z})^{r-1}
\end{array}\right], i, j \leq r-1
$$

is the same as in the treatment of $\mathbb{P}_{r-1}^{3}$ (with $k=r-1$ ).

- Two other groups

$$
\left[\begin{array}{c}
\tilde{x}^{i} \tilde{y}^{j}(1-\tilde{z})^{r} \\
0 \\
\tilde{x}^{i+1} \tilde{y}^{j}(1-\tilde{z})^{r}
\end{array}\right], \quad\left[\begin{array}{c}
0 \\
\tilde{x}^{j} \tilde{y}^{i}(1-\tilde{z})^{r} \\
\tilde{x}^{j} \tilde{y}^{i+1}(1-\tilde{z})^{r}
\end{array}\right], \quad i \leq r-1, j \leq r+1
$$

are the two last families of $\mathcal{B}_{r}$.

When similar computations are conducted for the two other families of functions of $\mathcal{S}_{r}$ :

$$
\left[\begin{array}{c}
x^{i} y^{j} z^{k+1} \\
0 \\
-x^{i+1} y^{j} z^{k}
\end{array}\right], \quad\left[\begin{array}{c}
x^{i} y^{j+1} z^{k} \\
-x^{i+1} y^{j} z^{k} \\
0
\end{array}\right], \quad k=r-1-i-j
$$

We obtain exactly the same form for $D F^{*} E$, only the expression of coefficients $b_{0}, b_{1}, \ldots, b_{9}$ being different. Therefore, we have shown that to generate $\mathcal{R}_{r}$, all the functions of $\mathcal{B}_{r}$ are necessary and sufficient.

Proposition 1. The dimensions of the optimal finite elements spaces are the following ones:

- Tetrahedron: $\operatorname{dim} \mathcal{R}_{r}=\frac{r(r+2)(r+3)}{2}$

- Hexahedron: $\operatorname{dim} \mathcal{Q}_{r}=3 r(r+2)^{2}$

- Prism: $\operatorname{dim} \mathcal{W}_{r}=\frac{r(r+2)(3 r+7)}{2}$

- Pyramid: $\operatorname{dim} \mathcal{B}_{r}=\frac{r(r+3)(2 r+3)}{2}$

Remark 1. The optimal finite element spaces $\hat{P}_{r}$ we constructed will be called "Optimal first family", since their construction relies on the inclusion of Nédélec's first family in the real space. The usual Nédélec's first family, denoted $\hat{P}_{r}^{1}$ consist of the following spaces:

- Tetrahedron:

$$
\hat{P}_{r}^{1}=\mathcal{R}_{r}
$$




\section{- Hexahedron:}

$$
\hat{P}_{r}^{1}=\mathbb{Q}_{r-1, r, r} \times \mathbb{Q}_{r, r-1, r} \times \mathbb{Q}_{r, r, r-1}
$$

- Prism:

$$
\hat{P}_{r}^{1}=\left(\mathcal{R}_{r}(\hat{x}, \hat{y}) \otimes \mathbb{P}_{r}(\hat{z})\right) \times\left(\mathbb{P}_{r}(\hat{x}, \hat{y}) \otimes \mathbb{P}_{r-1}(\hat{z})\right)
$$

- Pyramid:

$$
\begin{aligned}
& \hat{P}_{r}^{1}=\mathbb{B}_{r-1}^{3} \oplus\left\{\frac{\hat{x}^{p} \hat{y}^{p}}{(1-\hat{z})^{p+2}}\left[\begin{array}{c}
\hat{y}(1-\hat{z}) \\
\hat{x}(1-\hat{z}) \\
\hat{x} \hat{y}
\end{array}\right], 0 \leq p \leq r-1\right\} \\
& \oplus\left\{\frac{\hat{x}^{m} \hat{y}^{n+2}}{(1-\hat{z})^{m+2}}\left[\begin{array}{c}
(1-\hat{z}) \\
0 \\
\hat{x}
\end{array}\right], \frac{\hat{x}^{n+2} \hat{y}^{m}}{(1-\hat{z})^{m+2}}\left[\begin{array}{c}
0 \\
(1-\hat{z}) \\
\hat{y}
\end{array}\right], 0 \leq m \leq n \leq r-2\right\} \\
& \oplus\left\{\frac{\hat{x}^{p} \hat{y}^{q}}{(1-\hat{z})^{p+q+1-r}}\left[\begin{array}{c}
(1-\hat{z}) \\
0 \\
\hat{x}
\end{array}\right], \frac{\hat{x}^{q} \hat{y}^{p}}{(1-\hat{z})^{p+q+1-r}}\left[\begin{array}{c}
0 \\
(1-\hat{z}) \\
\hat{y}
\end{array}\right], \begin{array}{l}
0 \leq p \leq r-1 \\
0 \leq q \leq r
\end{array}\right\}
\end{aligned}
$$

Here, the finite element space we propose on the pyramid is issued from the optimal space by enforcing conformity with hexahedra of the first family. The dimension of this pyramidal space is equal to $\frac{r\left(2 r^{2}+9 r+5\right)}{2}$. Hexahedral and pyramidal spaces of the first family $\hat{P}_{r}^{1}$ need $(r+1)^{3}$ Gauss-Legendre-like quadrature points to ensure a positive definite mass matrix and keep the optimal order of convergence in $O\left(h^{r-1}\right)$ for deformed meshes, whereas optimal spaces $\hat{P}_{r}$ need $(r+2)^{3}$ quadrature points for a convergence in $O\left(h^{r}\right)$. Cubature rules used for pyramidal basis functions are the same formulas as described in (Bergot et al., 2010).

\section{H(curl) Conformity and Tangential Traces}

In the construction of these optimal finite element spaces, the $H(\mathrm{curl})$-conformity between elements has not been enforced but is nevertheless ensured, as the tangential restriction of the optimal finite element spaces is the following:

- Triangular faces: $\mathcal{R}_{r}(x, y)$

- Quadrilateral faces: $\mathbb{Q}_{r-1, r+1} \times \mathbb{Q}_{r+1, r-1}$

It should be noticed that the restriction on quadrilateral faces coincide with the optimal finite element space for edge elements in 2-D (see (Arnold et al., 2005)), and that optimal hexahedral finite element space is the same as the space found in (Falk et al., 2011) (for $r=1$ ). However, the construction proposed by Falk, Gatto and Monk is different from our construction, since it relies on the construction of facet elements (for the discretization of $\mathrm{H}(\mathrm{div})$ ). For pyramidal elements, since the $\mathrm{H}$ (curl) conformity is not immediate, we provide a proof of that.

Lemma 1. - If $p \in \mathbb{B}_{r}(\hat{K}), p \in H^{5 / 2-\varepsilon}(\hat{K})$ for any $\varepsilon>0$.

- If $p=\hat{x}^{i} \hat{y}^{j}(1-\hat{z})^{k-(i+j)}$, p belongs to $H^{3 / 2+k-\varepsilon}(\hat{K})$

with $H^{m}(\hat{K})$ the classical Sobolev space.

Proof. Let us take

$$
p=\hat{x}^{i} \hat{y}^{j}(1-\hat{z})^{k-(i+j)}
$$

For $m_{1}<i, m_{2}<j, i+j<k$, the $\mathrm{m}$-th derivative of $p$ is proportional to:

$$
\frac{\partial p}{\partial \hat{x}^{m_{1}} \partial \hat{y}^{m_{2}} \partial \hat{z}^{m_{3}}}=\alpha \hat{x}^{i-m_{1}} \hat{y}^{j-m_{2}}(1-\hat{z})^{k-i-j-m_{3}}
$$


Then when writing the integral in the cube:

$$
\begin{aligned}
\int_{\hat{K}}\left(\frac{\partial p}{\partial \hat{x}^{m_{1}} \partial \hat{y}^{m_{2}} \partial \hat{z}^{m_{3}}}\right)^{2} d \hat{x} d \hat{y} d \hat{z} & =\int_{[-1,1] \times[0,1]}(1-\tilde{z})^{2}\left(\frac{\partial p}{\partial \hat{x}^{m_{1}} \partial \hat{y}^{m_{2}} \partial \hat{z}^{m_{3}}}\right)^{2} d \tilde{x} d \tilde{y} d \tilde{z} \\
& =\alpha^{2} \int_{[-1,1] \times[0,1]}^{\tilde{x}^{2\left(i-m_{1}\right)} \tilde{y}^{2\left(j-m_{2}\right)}(1-\tilde{z})^{2\left(k-m_{1}-m_{2}-m_{3}+1\right)}}
\end{aligned}
$$

This integral is definite under the condition

$$
k+1-\left(m_{1}+m_{2}+m_{3}\right) \geq-\frac{1}{2}+\varepsilon \Leftrightarrow\left(m_{1}+m_{2}+m_{3}\right) \leq k+\frac{3}{2}-\varepsilon
$$

with $\varepsilon>0$. For any monomial $p$ of $\in \mathbb{B}_{r}(\hat{K})$, p is either constant or $p=\hat{x}^{i} \hat{y}^{j}(1-\hat{z})^{k-(i+j)}$ with $k \geq 1$. As a result, $\mathbb{B}_{r}(\hat{K}) \subset H^{5 / 2-\varepsilon}(\hat{K})$.

Theorem 2. With the optimal finite element spaces $P_{r}^{F}$ constructed in the previous section, we have

$$
V_{h}(\Omega) \subset H(\operatorname{curl}, \Omega) .
$$

Proof. - In a first place, we have to check that on each element $\mathrm{K}, P_{r}^{F} \subset H($ curl,$K)$. Let us consider $\mathbf{p} \in P_{r}^{F}$, we have:

$$
\begin{gathered}
\hat{p}=D F^{*} \mathbf{p} \circ F \\
\text { curl } \hat{p}=|D F| D F^{-1} \operatorname{curl} \mathbf{p} \circ F
\end{gathered}
$$

The transformation $F$ belongs to $H^{5 / 2-\varepsilon}(\hat{K})$ (with $\varepsilon>0$ ), and $D F$ belongs to $H^{3 / 2-\varepsilon}(\hat{K})$, by application of lemma (1). Therefore, it is equivalent to prove that $\hat{p}$ belongs to $H(\mathrm{curl}, \hat{K})$.

If $\hat{p} \in \mathbb{B}_{r-1}^{3}, \hat{p}$ belongs to $H^{5 / 2-\varepsilon}(\hat{K})$, then curl $\hat{p}$ belongs to $H^{3 / 2-\varepsilon}(\hat{K})$. For the other monomials of $\mathcal{B}_{r}$, two cases are distinguished:

$$
-\hat{p}=\left[\begin{array}{c}
\frac{\hat{y}}{1-\hat{z}} \\
\frac{\hat{x}}{1-\hat{z}} \\
\frac{\hat{x} \hat{y}}{(1-\hat{z})^{2}}
\end{array}\right]=\nabla\left(\frac{\hat{x} \hat{y}}{1-\hat{z}}\right)
$$

Since the curl of a gradient is null, we have

$$
\operatorname{curl} \hat{p}=0
$$

By applying lemma (1), $\hat{p} \in H^{3 / 2-\varepsilon}(\hat{K})$.

- Each component is of the form $\hat{p}_{n}=\hat{x}^{i} \hat{y}^{j}(1-\hat{z})^{k-i-j}$ with $i, j \leq k+1$ and $k \geq 1$. By application of lemma (1), $\hat{p} \in H^{5 / 2-\varepsilon}(\hat{K})$, and $\operatorname{curl} p \in H^{3 / 2-\varepsilon}(\hat{K})$.

As a result, $\mathcal{B}_{r} \subset H^{5 / 2-\varepsilon}(\hat{K})$ and $\operatorname{curl} \mathcal{B}_{r} \subset H^{3 / 2-\varepsilon}(\hat{K})$. It should also be noticed that the curl of each function of $\mathcal{B}_{r}$ is bounded in $\hat{K}$, even though the limit of $\operatorname{curl} p$ when $(\hat{x}, \hat{y}, \hat{z})$ tends to the apex of the pyramid is multi-valued.

- In a second place, we have to check that the tangential restriction of $\mathcal{B}_{r}$ is equal to $\mathcal{R}_{r}(x, z)$ for a triangular face, and to $\mathbb{Q}_{r-1, r+1} \times \mathbb{Q}_{r+1, r-1}$ for a quadrilateral face. The result is immediate for the quadrilateral face. Let us consider a triangular face $\hat{x}=(1-\hat{z})$, with $n=[1,0,1]$. A first step is to write $\mathcal{S}_{r}(\hat{y}, \hat{z})$ on this face.

We consider the transformation $f$ from the unit triangle $\hat{T}(\eta, \xi)$ to the triangular face $\hat{x}=1-\hat{z}$ of the pyramid $\hat{K}(\hat{x}, \hat{y}, \hat{z})$

$$
f=\mid \begin{aligned}
& \hat{x}=1-\xi \\
& \hat{y}=2 \eta+\xi-1 \\
& \hat{z}=\xi
\end{aligned}
$$


This transformation $f$ is a diffeomorphism from $\hat{T}$ to the face $\hat{x}=1-\hat{z}$. We have

$$
D f=\left[\begin{array}{cc}
0 & -1 \\
2 & 1 \\
0 & 1
\end{array}\right], \quad D f^{-*}=\frac{1}{4}\left[\begin{array}{cc}
1 & -2 \\
2 & 0 \\
-1 & 2
\end{array}\right]
$$

where $D f$ is the jacobian of transformation $f$ and $D f^{-*}$ the transpose of the pseudo-inverse of $D f$. We consider a monomial $\varphi \in \mathcal{S}_{r}(\eta, \xi)$ :

$$
\varphi(\eta, \xi)=\left[\begin{array}{c}
\eta^{i} \xi^{j+1} \\
-\eta^{i+1} \xi^{j}
\end{array}\right]
$$

with $i+j=r-1$. On the face $\hat{x}=1-\hat{z}$ of $\hat{K}, \varphi$ reads

$$
\varphi(\hat{x}, \hat{y}, \hat{z})=D f^{-*} \varphi(\eta, \xi) \circ f^{-1}=\left(\frac{\hat{y}+1-\hat{z}}{2}\right)^{i} \hat{z}^{j}\left[\begin{array}{c}
\hat{y}+1 \\
2 \hat{z} \\
-\hat{y}-1
\end{array}\right]
$$

One can add as many polynomials of $\mathbb{P}_{r-1}^{2}$ as we want, since the additional space $\mathcal{S}_{r}$ is searched $\left(\mathcal{R}_{r}=\right.$ $\left.\mathbb{P}_{r-1}^{2}+\mathcal{S}_{r}\right)$. We add $\left(t_{1}-2 t_{2}\right)\left(\frac{\hat{y}+1-\hat{z}}{2}\right)^{i} \hat{z}^{j}$ with

$$
t_{1}=\left[\begin{array}{c}
-1 \\
0 \\
1
\end{array}\right] \quad t_{2}=\left[\begin{array}{l}
0 \\
1 \\
0
\end{array}\right]
$$

Therefore, we obtain

$$
\mathcal{S}_{r}(\hat{y}, \hat{z})=\operatorname{Span}\left\{\left[\begin{array}{c}
\hat{y}^{j+1}(1-\hat{z})^{i} \\
-2 \hat{y}^{j}(1-\hat{z})^{i+1} \\
-\hat{y}^{j+1}(1-\hat{z})^{i}
\end{array}\right], \quad i+j=r-1\right\}
$$

Let us now compute the tangential restrictions of $\mathcal{B}_{r}$, that is $p_{n}(\hat{y}, \hat{z})=(p \times n) \times n_{\mid \hat{x}=(1-\hat{z})}$

- If $p \in \mathbb{B}_{r-1}^{3}$, we have immediatly $p_{n} \in \mathbb{P}_{r-1}^{2}(\hat{y}, \hat{z})$.

- Otherwise, for $0 \leq i \leq r-1$

$$
p=\left[\begin{array}{c}
\frac{\hat{x}^{i} \hat{y}^{i+1}}{(1-\hat{z})^{i+1}} \\
\frac{\hat{x}^{i+1} \hat{y}^{i}}{(1-\hat{z})^{i+1}} \\
\frac{\hat{x}^{i+1} \hat{y}^{i+1}}{(1-\hat{z})^{i+2}}
\end{array}\right] \Longrightarrow p_{n}=\left[\begin{array}{c}
0 \\
\hat{y}^{i} \\
0
\end{array}\right] \in \mathbb{P}_{r-1}^{2}(\hat{y}, \hat{z})
$$

For $0 \leq m \leq n \leq r-2$,

$$
\begin{aligned}
& p=\left[\begin{array}{c}
\frac{\hat{x}^{m} \hat{y}^{n+2}}{(1-\hat{z})^{m+1}} \\
0 \\
\hat{x}^{m+1} \hat{y}^{n+2} \\
(1-\hat{z})^{m+2}
\end{array}\right] \quad \Longrightarrow \quad p_{n}=\left[\begin{array}{l}
0 \\
0 \\
0
\end{array}\right] \\
& p=\left[\begin{array}{c}
0 \\
\frac{\hat{x}^{n+2} \hat{y}^{m}}{(1-\hat{z})^{m+1}} \\
\hat{x}^{n+2} \hat{y}^{m+1} \\
(1-\hat{z})^{m+2}
\end{array}\right] \Longrightarrow p_{n}=\left[\begin{array}{c}
\hat{y}^{m+1}(1-\hat{z})^{n-m} \\
-2 \hat{y}^{m}(1-\hat{z})^{n-m+1} \\
-\hat{y}^{m+1}(1-\hat{z})^{n-m}
\end{array}\right] \in \mathbb{P}_{r-1}^{2}(\hat{y}, \hat{z})
\end{aligned}
$$


For $0 \leq p \leq r-1,0 \leq q \leq r+1$

$$
\begin{aligned}
& p=\left[\begin{array}{c}
\frac{\hat{x}^{p} \hat{y}^{q}}{(1-\hat{z})^{p+q-r}} \\
0 \\
\frac{\hat{x}^{p+1} \hat{y}^{q}}{(1-\hat{z})^{p+q+1-r}}
\end{array}\right] \Longrightarrow p_{n}=\left[\begin{array}{l}
0 \\
0 \\
0
\end{array}\right] \\
& p=\left[\begin{array}{c}
0 \\
\frac{\hat{x}^{q} \hat{y}^{p}}{(1-\hat{z})^{p+q-r}} \\
\frac{\hat{x}^{q} \hat{y}^{p+1}}{(1-\hat{z})^{p+q+1-r}}
\end{array}\right] \Longrightarrow p_{n}=\left[\begin{array}{c}
\hat{y}^{p+1}(1-\hat{z})^{r-p-1} \\
-2 \hat{y}^{p}(1-\hat{z})^{r-p} \\
-\hat{y}^{p+1}(1-\hat{z})^{r-p-1}
\end{array}\right] \in \mathcal{S}_{r}(\hat{y}, \hat{z})
\end{aligned}
$$

The same result can be obtained with other triangular faces.

By considering dimension of the spaces, we obtain

$$
\begin{aligned}
\hat{P}_{r \mid \hat{x}=1-\hat{z} \text { ou } \hat{x}=\hat{z}-1} & =\mathcal{R}_{r}(\hat{y}, \hat{z}) \\
\hat{P}_{r \mid \hat{y}=1-\hat{z} \text { ou } \hat{y}=\hat{z}-1} & =\mathcal{R}_{r}(\hat{x}, \hat{z}) \\
\hat{P}_{r \mid \hat{z}=0} & =\mathbb{Q}_{r-1, r+1}(\hat{x}, \hat{y}) \times \mathbb{Q}_{r+1, r-1}(\hat{x}, \hat{y})
\end{aligned}
$$

These restrictions are the same as the restrictions obtained with hexahedra, prisms and tetrahedra.

\section{Stability}

The stability for $H(\mathrm{curl})$-conforming elements for Maxwell's equations comes from the exact sequence of De Rham diagram (see (Monk, 2002)), that is the approximation spaces must verify

$$
\begin{aligned}
& \operatorname{Im} \operatorname{grad} W_{r+1}^{1}=\operatorname{Ker} W_{r}^{r o t}=\left\{u \in W_{r}^{\text {rot }} \mid \text { curl } u=0\right\} \\
& \operatorname{Im} \text { curl } W_{r}^{\text {curl }}=\operatorname{Ker} W_{r-1}^{\text {div }}=\left\{u \in W_{r-1}^{\text {div }} \mid \text { div } u=0\right\} \\
& \operatorname{Im} \operatorname{div} W_{r-1}^{\text {div }}=\operatorname{Ker} W_{r-2}^{2}=W_{r-2}^{2}
\end{aligned}
$$

where $W_{r}^{H 1}, W_{r}^{c u r l}, W_{r}^{\text {div }}$ and $W_{r}^{2}$ are the spaces of order $r$ discretizing respectively $H^{1}(\Omega), H($ curl,$\Omega), H(d i v, \Omega)$ and $L^{2}(\Omega)$.

The whole sequence will be detailed in a future work presenting the $H(d i v)$-conforming elements but we have checked that with the spaces for $H^{1}$ approximation presented in (Bergot et al., 2010) and the present spaces for $H($ curl) approximation, we have

$$
\begin{aligned}
& \operatorname{grad} \hat{P}_{r}^{H^{1}} \subset \hat{P}_{r}^{H(\text { curl })} \\
& \operatorname{grad} \hat{P}_{r}^{H^{1}} \subset \hat{P}_{r}^{1}
\end{aligned}
$$

The proof will be detailed in a future work. Besides, we numerically show that the sequence is exact

$$
\begin{aligned}
& \operatorname{Im} \operatorname{grad} \hat{P}_{r}^{H^{1}}=\operatorname{Ker} \hat{P}_{r}^{H(\text { curl })} \\
& \operatorname{Im} \operatorname{grad} \hat{P}_{r}^{H^{1}}=\operatorname{Ker} \hat{P}_{r}^{1}
\end{aligned}
$$

by comparing the dimension of the space $\operatorname{grad} \hat{P}_{r}^{H^{1}}$ and the dimension ot the kernel of the stiffness matrix. 


\section{Construction of the Basis Functions}

\subsection{Nodal Basis Functions}

Nodal basis functions are popular for continuous finite elements discretizing $H^{1}(\Omega)$, but are less known for edge elements. Let us mention the works of Graglia (Graglia et al., 1997) for tetrahedra and the works of Cohen \& Monk (Cohen and Monk, 1998) for hexahedra. For edge elements, each degree of freedom is associated with a position and a direction. If $\left(\xi_{i}\right)_{1 \leq i \leq r+1}$ are Gauss-Lobatto points, the points $\left(\xi_{1}^{I}, \xi_{2}^{I}, \xi_{3}^{I}\right)=\left(\xi_{2}, \xi_{3}, \ldots, \xi_{r}\right)$ will be called interior Gauss-Lobatto points. For nodal points of the triangle, we will choose electrostatic points as described in (Hesthaven and Teng, 2000). Let us detail the positions and directions of the degrees of freedom for the different elements:

Hexahedron : As shown in figure Fig. 2 a tensor product between Gauss-Lobatto points and interior Gauss-Lobatto points is considered, more precisely, three families of degrees of freedom:

$$
\begin{array}{ll}
\left(\xi_{i}^{I}, \xi_{j}, \xi_{k}\right) \text { with direction } e_{x}, & 1 \leq i \leq r, 1 \leq j, k \leq r+2 \\
\left(\xi_{j}, \xi_{i}^{I}, \xi_{k}\right) \text { with direction } e_{y}, & 1 \leq i \leq r, 1 \leq j, k \leq r+2 \\
\left(\xi_{j}, \xi_{k}, \xi_{i}^{I}\right) \text { with direction } e_{z}, & 1 \leq i \leq r, 1 \leq j, k \leq r+2
\end{array}
$$

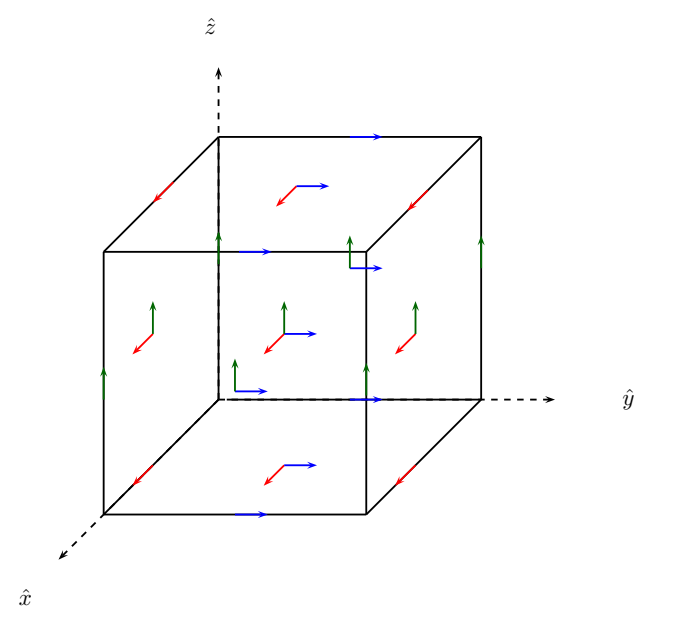

Figure 2: Location of degrees of freedom for first-order hexahedron

Tetrahedron : As displayed in figure Fig 3 for first-order tetrahedra, degrees of freedom are placed as follows

- For the edges, degrees of freedom are placed on interior Gauss-Lobatto points

- For the faces, two degrees of freedom are placed on each interior point of a nodal triangle of order $\mathrm{r}+1$

- For the volume, three degrees of freedom are placed on each interior point of a nodal tetrahedron of order $\mathrm{r}+1$ (nodal points are chosen as in (Hesthaven and Teng, 2000)).

Prisms : As shown in figure Fig. 4 for the first-order prism, degrees of freedom along $(\hat{x}, \hat{y})$ are chosen as the tensorial product between a triangle equal to the triangular face of a tetrahedron and Gauss-Lobatto points. For the degrees of freedom oriented along $\hat{z}$, they are equal to the tensorial product of a nodal triangle of order $\mathrm{r}+1$ and interior Gauss-Lobatto points. 


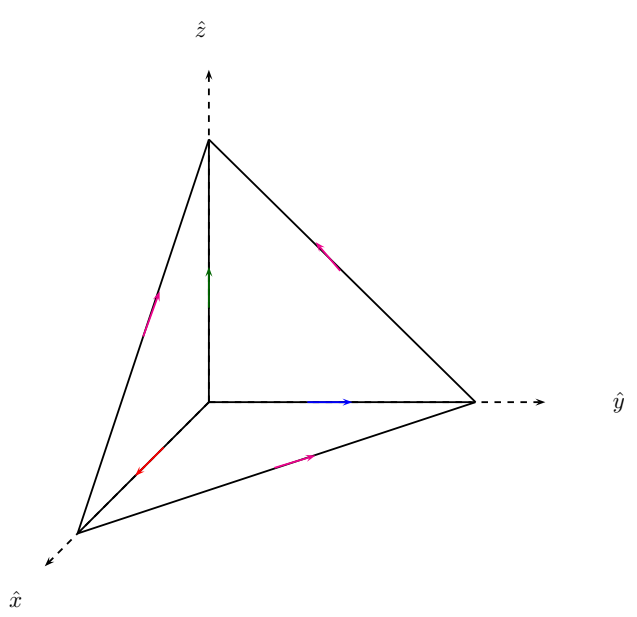

Figure 3: Location of degrees of freedom for first-order tetrahedron

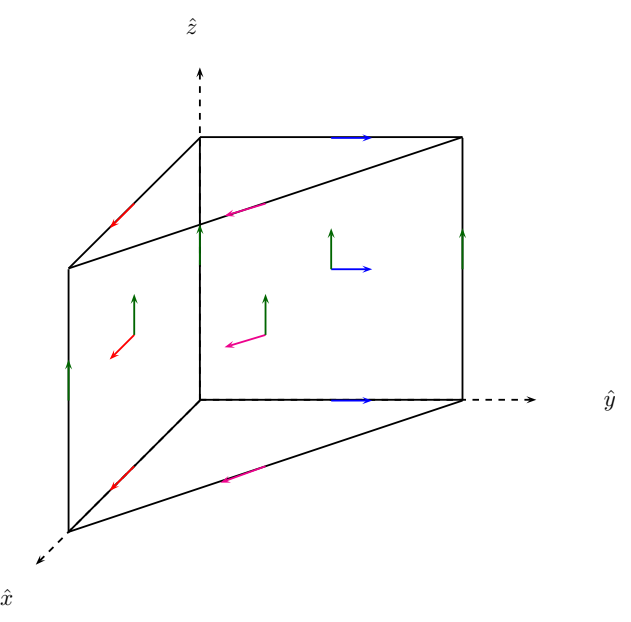

Figure 4: Location of degrees of freedom for first-order prism

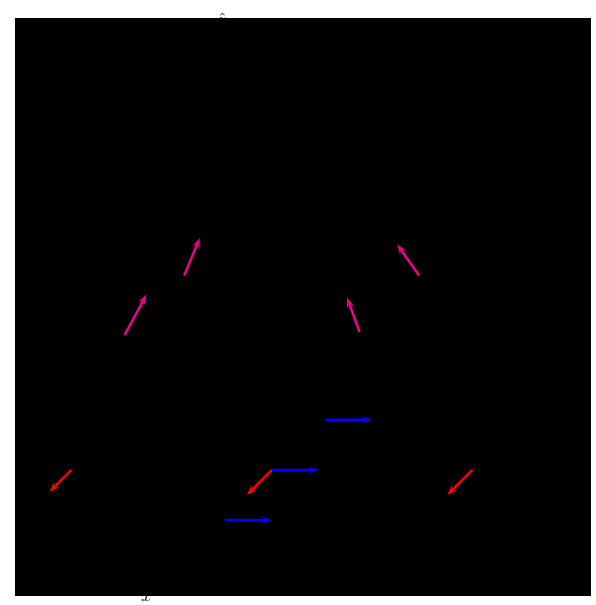

Figure 5: Location of degrees of freedom for first-order pyramid 
Pyramids : As displayed in figure Fig. 5 for the first-order pyramid, degrees of freedom on the quadrilateral base are placed in the same way as a face of an hexahedron, degrees of freedom on the triangular faces are placed similarly to a face of a tetrahedron. Three degrees of freedom are associated with each interior point of a nodal pyramid of order $\mathrm{r}+1$. The location of nodal points on the pyramid is detailed in (Bergot et al., 2010).

The nodal basis functions $\hat{\varphi}$ are defined as follows:

Definition 3. Let $\left(\hat{M}_{i}\right)$ be the position of degrees of freeedom and $\hat{t}_{i}$ the direction associated with each degree of freedom, and $\left(\psi_{i}\right)_{1 \leq i \leq n_{r}}$ a basis of the finite element space $\hat{P}_{r}$. The Vandermonde matrix is equal to:

$$
V D M_{i, j}=\hat{\psi}_{i}\left(\hat{M}_{j}\right) \cdot \hat{t}_{j}, \quad 1 \leq i, j \leq n_{r}
$$

The basis function $\hat{\varphi}_{i}$ associated with the point $\hat{M}_{i}$ is defined by the relation

$$
\hat{\varphi}_{i}=\sum_{1 \leq j \leq n_{r}}\left(V D M^{-1}\right)_{i, j} \hat{\psi}_{j}
$$

Remark 2. The points $\hat{M}_{i}$ are specified with an orientation $\hat{t}_{i}$, but can correspond to a same geometric point (at most three degrees of freedom per geometric point).

Remark 3. The invertibility of Vandermonde matrix is an open issue, but with our choice of degrees of freedom, we have observed that this matrix is invertible, therefore the finite element is unisolvent.

The main drawback of nodal basis functions is the bad condition number of Vandermonde matrix, that can lead to important round-off errors in the computations. The figure Fig 6 shows the condition number of Vandermonde matrix for the optimal first family and first family in the case of tetrahedra, prisms and pyramids. We notice that the prism uses actually the Vandermonde matrix of a triangle, which contains much less degrees of freedom than tetrahedra. As a result the condition number of Vandermonde matrix for triangles is quite small.

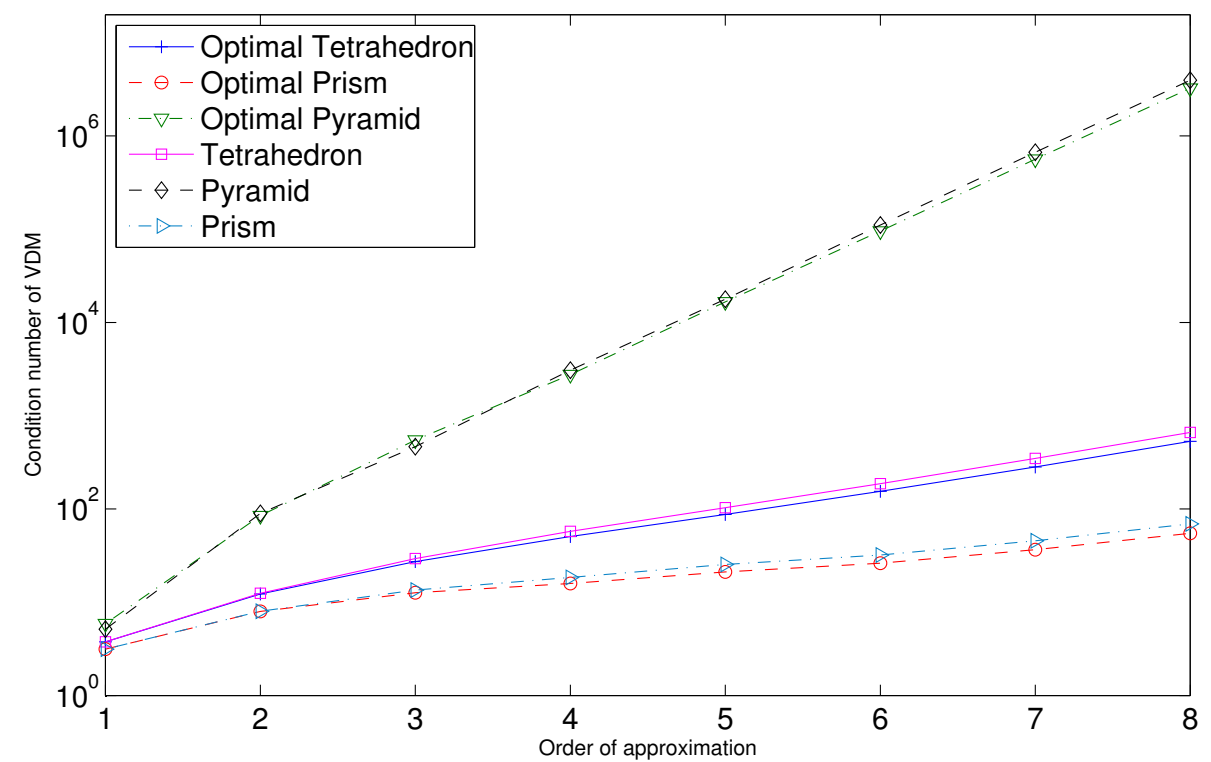

Figure 6: Condition number of Vandermonde matrix versus the order of approximation for first family and optimal first family for the tetrahedron, pyramid and prism. 
It can be noticed that the condition number is not very different for the two families, the optimal finite element spaces providing a better condition number. For the first family $\hat{P}_{r}^{1}$, Gauss points have been chosen instead of interior Gauss-Lobatto points. We remark that basis functions $\psi_{i}$ chosen for the pyramid do not provide a good conditioning, which is problematic for $r \geq 5$. The basis functions $\psi_{i}$ expressed on the cube chosen for the pyramid are the following ones:

$$
\begin{aligned}
& \left\{\left[\begin{array}{c}
P_{i, j, k} \\
0 \\
0
\end{array}\right],\left[\begin{array}{c}
0 \\
P_{i, j, k} \\
0
\end{array}\right],\left[\begin{array}{c}
0 \\
0 \\
P_{i, j, k}
\end{array}\right], \begin{array}{l}
0 \leq i, j \leq r-1 \\
0 \leq k \leq r-1-\max (i, j)
\end{array}\right\} \\
& \left\{P_{p}^{0,0}(\tilde{x}) P_{p}^{0,0}(\tilde{y})(1-\tilde{z})^{p}\left[\begin{array}{c}
\tilde{y} \\
\tilde{x} \\
\tilde{x} \tilde{y}
\end{array}\right], 0 \leq p \leq r-1\right\} \\
& \left\{P_{m}^{0,0}(\tilde{x}) P_{n+2}^{0,0}(\tilde{y})(1-\tilde{z})^{n+1}\left[\begin{array}{l}
1 \\
0 \\
\tilde{x}
\end{array}\right], P_{n+2}^{0,0}(\tilde{x}) P_{m}^{0,0}(\tilde{y})(1-\tilde{z})^{n+1}\left[\begin{array}{l}
0 \\
1 \\
\tilde{y}
\end{array}\right], 0 \leq m \leq n \leq r-2\right\} \\
& \left\{P_{p}^{0,0}(\tilde{x}) P_{q}^{0,0}(\tilde{y})(1-\tilde{z})^{r}\left[\begin{array}{c}
1 \\
0 \\
\tilde{x}
\end{array}\right], P_{q}^{0,0}(\tilde{x}) P_{p}^{0,0}(\tilde{y})(1-\tilde{z})^{r}\left[\begin{array}{c}
0 \\
1 \\
\tilde{y}
\end{array}\right], \begin{array}{l}
0 \leq p \leq r-1 \\
0 \leq q \leq r+1
\end{array}\right\}
\end{aligned}
$$

with

$$
\begin{gathered}
\tilde{x}=\frac{\hat{x}}{1-\hat{z}}, \quad \tilde{y}=\frac{\hat{y}}{1-\hat{z}} \quad \tilde{z}=\hat{z} \\
P_{i, j, k}=P_{i}^{0,0}(\tilde{x}) P_{j}^{0,0}(\tilde{y}) P_{k}^{2 \max (i, j)+2,0}(2 \tilde{z}-1)(1-\tilde{z})^{\max (i, j)}
\end{gathered}
$$

This basis is written by using Jacobi polynomials $P_{n}^{\alpha, \beta}$ which are orthogonal with respect to weight $(1-x)^{\alpha}(1+x)^{\beta}$. In order to reduce the condition number, each basis function $\psi_{i}$ is normalized so that $\left\|\psi_{i}\right\|_{L^{2}(\hat{K})}=1$.

\subsection{Hierarchical Basis Functions}

Hierarchical basis functions are more popular in finite elements since they are often written with a tensorial structure in the cube, and they do not need the inversion of a Vandermonde matrix. Therefore, we present a simple hierarchical base for the optimal first family. Jacobi polynomials $P_{i, j}^{\alpha, \beta}$ are used, but other choices could be considered such as integrated Lobatto-shape functions (see (Šolín et al., 2003)). Other sets of basis functions can also be constructed (e.g. (Zaglmayr, 2006)), in order to provide some attrative properties such as highly sparse elementary matrices (see (Beuchler et al., 2011)) or well-conditioned mass and stiffness matrices (see (Xin et al., 2011)).

Proposition 2. The following basis functions are an hierarchical basis $H\left(\right.$ curl) conforming of $\hat{P}_{r}$

- Hexahedron: We consider the following parameters (each parameter being associated with a face of the cube)

$$
\left\{\begin{array} { l } 
{ \lambda _ { 1 } = \hat { x } } \\
{ \lambda _ { 2 } = \hat { y } } \\
{ \lambda _ { 3 } = \hat { z } }
\end{array} \quad \left\{\begin{array}{l}
\lambda_{4}=1-\hat{x} \\
\lambda_{5}=1-\hat{y} \\
\lambda_{6}=1-\hat{z}
\end{array}\right.\right.
$$




\section{HIERARCHICAL H(CURL) FUNCTIONS FOR THE CUBE}

For an edge $a:$ Let $a_{1}$ and $a_{2}$ the two faces that do not contain any vertex of a If $a$ is oriented along $e_{x}$

If a is oriented along $e_{y}$

$$
\left[\begin{array}{c}
\lambda_{a_{1}} \lambda_{a_{2}} \\
0 \\
0
\end{array}\right] P_{i}^{0,0}(2 \hat{x}-1), \quad 0 \leq i \leq r-1
$$

$$
\left[\begin{array}{c}
0 \\
\lambda_{a_{1}} \lambda_{a_{2}} \\
0
\end{array}\right] P_{i}^{0,0}(2 \hat{y}-1), \quad 0 \leq i \leq r-1
$$

If a is oriented along $e_{z}$

$$
\left[\begin{array}{c}
0 \\
0 \\
\lambda_{a_{1}} \lambda_{a_{2}}
\end{array}\right] P_{i}^{0,0}(2 \hat{z}-1), \quad 0 \leq i \leq r-1
$$

For a face $f$ : Let $f_{1}$ be the face directly opposite to $f$

If $f$ is in the plane $\left(e_{x}, e_{y}\right)$

$$
\begin{aligned}
& {\left[\begin{array}{c}
\lambda_{2} \lambda_{5} \lambda_{f_{1}} \\
0 \\
0
\end{array}\right] P_{i}^{0,0}(2 \hat{x}-1) P_{j}^{1,1}(2 \hat{y}-1)} \\
& {\left[\begin{array}{c}
0 \\
\lambda_{1} \lambda_{4} \lambda_{f_{1}} \\
0
\end{array}\right] P_{j}^{1,1}(2 \hat{x}-1) P_{i}^{0,0}(2 \hat{y}-1)} \\
& {\left[\begin{array}{l}
0 \leq i, j \leq r-1 \\
\end{array}\right.}
\end{aligned}
$$

If $f$ is in the plane $\left(e_{y}, e_{z}\right)$

$$
\begin{aligned}
& {\left[\begin{array}{c}
0 \\
\lambda_{3} \lambda_{6} \lambda_{f_{1}} \\
0
\end{array}\right] P_{i}^{0,0}(2 \hat{y}-1) P_{j}^{1,1}(2 \hat{z}-1)} \\
& {\left[\begin{array}{c}
0 \\
0 \\
\lambda_{2} \lambda_{5} \lambda_{f_{1}}
\end{array}\right] P_{j}^{1,1}(2 \hat{y}-1) P_{i}^{0,0}(2 \hat{z}-1)}
\end{aligned}
$$

If $f$ is in the plane $\left(e_{x}, e_{z}\right)$

$$
\begin{aligned}
& {\left[\begin{array}{c}
\lambda_{3} \lambda_{6} \lambda_{f_{1}} \\
0 \\
0
\end{array}\right] P_{i}^{0,0}(2 \hat{x}-1) P_{j}^{1,1}(2 \hat{z}-1)} \\
& {\left[\begin{array}{c}
0 \\
0 \\
\lambda_{1} \lambda_{4} \lambda_{f_{1}}
\end{array}\right] P_{j}^{1,1}(2 \hat{x}-1) P_{i}^{0,0}(2 \hat{z}-1)}
\end{aligned}
$$

For the interior functions :

$$
\begin{aligned}
& {\left[\begin{array}{c}
\lambda_{2} \lambda_{3} \lambda_{5} \lambda_{6} \\
0 \\
0
\end{array}\right] P_{k}^{0,0}(2 \hat{x}-1) P_{i}^{1,1}(2 \hat{y}-1) P_{j}^{1,1}(2 \hat{z}-1)} \\
& {\left[\begin{array}{c}
0 \\
\lambda_{1} \lambda_{3} \lambda_{4} \lambda_{6} \\
0
\end{array}\right] P_{i}^{1,1}(2 \hat{x}-1) P_{k}^{0,0}(2 \hat{y}-1) P_{j}^{1,1}(2 \hat{z}-1) \quad 0 \leq i, j, k \leq r-1} \\
& {\left[\begin{array}{c}
0 \\
0 \\
\lambda_{1} \lambda_{2} \lambda_{4} \lambda_{5}
\end{array}\right] P_{i}^{1,1}(2 \hat{x}-1) P_{j}^{1,1}(2 \hat{y}-1) P_{k}^{0,0}(2 \hat{z}-1)}
\end{aligned}
$$


- Triangular prism: We consider the following parameters (each parameter being associated with a face). The parameters $\lambda$ are also associated with vertices of a triangular face.

$$
\left\{\begin{array} { l } 
{ \lambda _ { 1 } = \lambda _ { 4 } = 1 - \hat { x } - \hat { y } } \\
{ \lambda _ { 2 } = \lambda _ { 5 } = \hat { x } } \\
{ \lambda _ { 3 } = \lambda _ { 6 } = \hat { y } }
\end{array} \quad \left\{\begin{array}{l}
\beta_{1}=1-\hat{z} \\
\beta_{2}=\hat{z}
\end{array}\right.\right.
$$

\section{HIERARCHICAL H(CURL) FUNCTIONS FOR THE PRISM}

For an horizontal edge $a:$ Let $a_{1}$ and $a_{2}$ the two extremities of the edge $a$, and $f^{\prime}$ the horizontal opposite face

$$
\left(\lambda_{a_{1}} \nabla \lambda_{a_{2}}-\lambda_{a_{2}} \nabla \lambda_{a_{1}}\right) \beta_{f^{\prime}} P_{i}^{0,0}\left(\lambda_{a_{2}}-\lambda_{a_{1}}\right), \quad 0 \leq i \leq r-1, \quad 1 \leq a \leq 6
$$

For a vertical edge $a:$ Let $a_{1}$ the face that does not contain any vertex of $a$

$$
\left[\begin{array}{c}
0 \\
0 \\
\lambda_{a_{1}}
\end{array}\right] P_{i}^{0,0}\left(\beta_{2}-\beta_{1}\right), \quad 0 \leq i \leq r-1, \quad 1 \leq a \leq 3
$$

For a quadrilateral face $f:$ Let $\left[a_{1}, a_{2}\right]$ an edge in common with a triangular face $f^{\prime}$, and $f_{1}, f_{2}$ the two other quadrilateral faces

$$
\begin{gathered}
\left(\lambda_{a_{1}} \nabla \lambda_{a_{2}}-\lambda_{a_{2}} \nabla \lambda_{a_{1}}\right) \beta_{1} \beta_{2} P_{i}^{0,0}\left(\lambda_{a_{2}}-\lambda_{a_{1}}\right) P_{j}^{1,1}(2 \hat{z}-1) \\
\quad\left[\begin{array}{c}
0 \\
0 \\
\lambda_{a_{1}} \lambda_{a_{2}}
\end{array}\right] P_{j}^{1,1}\left(\lambda_{a_{2}}-\lambda_{a_{1}}\right) P_{i}^{0,0}(2 \hat{z}-1) \\
\end{gathered}
$$

For a triangular face $f:$ Let $\left[a_{1}, a_{2}\right]$ and $\left[a_{1}, a_{3}\right]$ two edges of the triangular, $f_{1}$ and $f_{2}$ the associated quadrilateral faces, and $f^{\prime}$ the opposite triangular face

$$
\begin{gathered}
\left(\lambda_{a_{1}} \nabla \lambda_{a_{2}}-\lambda_{a_{2}} \nabla \lambda_{a_{1}}\right) \lambda_{f_{1}} \beta_{f^{\prime}} P_{i}^{0,0}\left(\lambda_{a_{2}}-\lambda_{a_{1}}\right) P_{j}^{0,0}\left(\lambda_{a_{3}}-\lambda_{a_{1}}\right) \\
\left(\lambda_{a_{1}} \nabla \lambda_{a_{3}}-\lambda_{a_{3}} \nabla \lambda_{a_{1}}\right) \lambda_{f_{2}} \beta_{f^{\prime}} P_{i}^{0,0}\left(\lambda_{a_{2}}-\lambda_{a_{1}}\right) P_{j}^{0,0}\left(\lambda_{a_{3}}-\lambda_{a_{1}}\right) \\
i, j \geq 0,0 \leq i+j \leq r-2
\end{gathered}
$$

For interior functions :

$$
\begin{aligned}
& \left(\lambda_{2} \nabla \lambda_{3}-\lambda_{3} \nabla \lambda_{2}\right) \lambda_{1} \beta_{1} \beta_{2} P_{i j k}(\hat{x}, \hat{y}, \hat{z}) \\
& \left(\lambda_{1} \nabla \lambda_{3}-\lambda_{3} \nabla \lambda_{1}\right) \lambda_{2} \beta_{1} \beta_{2} P_{i j k}(\hat{x}, \hat{y}, \hat{z}) \quad i, j \geq 0
\end{aligned}
$$

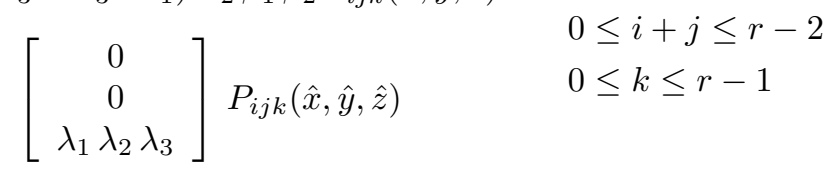

avec

$$
P_{i j k}(\hat{x}, \hat{y}, \hat{z})=P_{i}^{0,0}\left(\frac{2 \hat{x}}{1-\hat{y}}-1\right)(1-\hat{y})^{i} P_{j}^{2 i+1,0}(2 \hat{y}-1) P_{k}^{0,0}(2 \hat{z}-1)
$$


- Pyramid: We consider the following parameters:

$$
\left\{\begin{array} { l } 
{ \beta _ { 1 } = \frac { 1 - \hat { x } - \hat { z } } { 2 } } \\
{ \beta _ { 2 } = \frac { 1 - \hat { y } - \hat { z } } { 2 } } \\
{ \beta _ { 3 } = \frac { 1 + \hat { x } - \hat { z } } { 2 } } \\
{ \beta _ { 4 } = \frac { 1 + \hat { y } - \hat { z } } { 2 } }
\end{array} \left\{\begin{array} { l } 
{ \lambda _ { 1 } = \frac { \beta _ { 1 } \beta _ { 2 } } { 1 - \hat { z } } } \\
{ \lambda _ { 2 } = \frac { \beta _ { 2 } \beta _ { 3 } } { 1 - \hat { z } } } \\
{ \lambda _ { 3 } = \frac { \beta _ { 3 } \beta _ { 4 } } { 1 - \hat { z } } } \\
{ \lambda _ { 4 } = \frac { \beta _ { 4 } \beta _ { 1 } } { 1 - \hat { z } } } \\
{ \lambda _ { 5 } = \hat { z } } \\
{ \delta _ { 1 } = \delta _ { 3 } = \hat { x } } \\
{ \delta _ { 2 } = \delta _ { 4 } = \hat { y } }
\end{array} \quad \left\{\begin{array}{l}
\gamma_{1}=\frac{2 \hat{z}+\hat{x}+\hat{y}}{2} \\
\gamma_{3}=\frac{2 \hat{z}-\hat{x}-\hat{y}}{2} \\
\gamma_{4}=\frac{2 \hat{z}+\hat{x}-\hat{y}}{2}
\end{array}\right.\right.\right.
$$

Parameters $\beta_{i}$ are associated with triangular faces, since $\beta_{i}=0$ is the equation of a triangular face $i$. Parameters $\lambda_{i}$ are associated with vertices, since $\lambda_{i}\left(S_{j}\right)=\delta_{i, j}, S_{j}$ being the vertices. Parameters $\gamma_{i}$ are parametrizations of vertical edges, and $\delta_{i}$ parametrizations of horizontal edges. 


\section{H(CURL) FUNCTIONS FOR THE PYRAMID}

For an horizontal edge $a:$ The edge is oriented from vertex $a_{1}$ to vertex $a_{2}$, and the adjacent horizontal edges are $\left[a_{1}, a_{4}\right]$ et $\left[a_{2}, a_{3}\right]$

$$
\left(\lambda_{a_{1}} \nabla\left(\lambda_{a_{2}}+\lambda_{a_{3}}\right)-\lambda_{a_{2}} \nabla\left(\lambda_{a_{1}}+\lambda_{a_{4}}\right)\right) P_{i}^{0,0}\left(\delta_{a}\right), \quad 0 \leq i \leq r-1
$$

For a vertical edge $a:$ Let $s$ the vertex of a belonging to the base

$$
\left(\lambda_{s} \nabla \lambda_{5}-\lambda_{5} \nabla \lambda_{s}\right) P_{i}^{0,0}\left(\gamma_{s}\right), \quad 0 \leq i \leq r-1, \quad 1 \leq a \leq 4
$$

For the base :

$$
\begin{gathered}
\left(\lambda_{1} \nabla\left(\lambda_{2}+\lambda_{3}\right)-\lambda_{2} \nabla\left(\lambda_{1}+\lambda_{4}\right)\right) \beta_{4} P_{i}^{0,0}\left(\frac{\beta_{3}-\beta_{1}}{1-\hat{z}}\right) P_{j}^{1,1}\left(\frac{\beta_{4}-\beta_{2}}{1-\hat{z}}\right)(1-\hat{z})^{\max (i, j)-1} \\
\left(\lambda_{1} \nabla\left(\lambda_{3}+\lambda_{4}\right)-\lambda_{4} \nabla\left(\lambda_{2}+\lambda_{1}\right)\right) \beta_{3} P_{j}^{1,1}\left(\frac{\beta_{3}-\beta_{1}}{1-\hat{z}}\right) P_{i}^{0,0}\left(\frac{\beta_{4}-\beta_{2}}{1-\hat{z}}\right)(1-\hat{z})^{\max (i, j)-1} \\
0 \leq i, j \leq r-1
\end{gathered}
$$

For a triangular face $f:$ Let $\left[a_{1}, a_{2}\right]$ the horizontal edge, and $\left[a_{1}, a_{4}\right]$ et $\left[a_{2}, a_{3}\right]$ the two adjacent edges, and $f_{1}$ the triangular face of base $\left[a_{1}, a_{4}\right]$

$$
\begin{array}{cc}
\left(\lambda_{a_{2}} \nabla\left(\lambda_{a_{1}}+\lambda_{a_{4}}\right)-\lambda_{a_{1}} \nabla\left(\lambda_{a_{2}}+\lambda_{a_{3}}\right)\right) \lambda_{5} P_{i}^{0,0}\left(\delta_{f}\right) P_{j}^{0,0}\left(\gamma_{a_{1}}\right) & 0 \leq i+j \leq r-2 \\
\left(\lambda_{a_{1}} \nabla \lambda_{5}-\lambda_{5} \nabla \lambda_{a_{1}}\right) \beta_{f_{1}} P_{i}^{0,0}\left(\delta_{f}\right) P_{j}^{0,0}\left(\gamma_{a_{1}}\right) &
\end{array}
$$

For interior functions :

$$
\begin{array}{cl}
\left(\lambda_{1} \nabla\left(\lambda_{2}+\lambda_{3}\right)-\lambda_{2} \nabla\left(\lambda_{1}+\lambda_{4}\right)\right) \beta_{4} \lambda_{5} P_{i j k}(\hat{x}, \hat{y}, \hat{z}) & \\
\left(\lambda_{1} \nabla\left(\lambda_{3}+\lambda_{4}\right)-\lambda_{4} \nabla\left(\lambda_{2}+\lambda_{1}\right)\right) \beta_{3} \lambda_{5} P_{i j k}(\hat{x}, \hat{y}, \hat{z}) & 0 \leq i, j \leq r-2, \\
\left(\lambda_{1} \nabla \lambda_{5}-\lambda_{5} \nabla \lambda_{1}\right) \beta_{3} \beta_{4} P_{i j k}(\hat{x}, \hat{y}, \hat{z}) & 0 \leq k \leq r-2-\max (i, j)
\end{array}
$$

with

$$
P_{i j k}(\hat{x}, \hat{y}, \hat{z})=P_{i}^{0,0}\left(\frac{\beta_{3}-\beta_{1}}{1-\hat{z}}\right) P_{j}^{0,0}\left(\frac{\beta_{4}-\beta_{2}}{1-\hat{z}}\right) P_{k}^{2 \max (i, j)+2,0}(2 \hat{z}-1)(1-\hat{z})^{\max (i, j)-1}
$$


- Tetrahedron: We consider the following parameters

$$
\left\{\begin{array}{l}
\lambda_{1}=1-\hat{x}-\hat{y}-\hat{z} \\
\lambda_{2}=\hat{x} \\
\lambda_{3}=\hat{y} \\
\lambda_{4}=\hat{z}
\end{array}\right.
$$

The parameters $\lambda_{i}$ are associated with faces, since $\lambda_{i}=0$ is the equation of face $i$. They are also associated with vertices since $\lambda_{i}\left(S_{j}\right)=\delta_{i, j}$, $S_{i}$ being the vertices.

\section{HIERARCHICAL H(CURL) FUNCTIONS FOR A TETRAHEDRON}

For an edge $a:$ The edge is oriented from vertex $a_{1}$ to vertex $a_{2}$

$$
\left(\lambda_{a_{1}} \nabla \lambda_{a_{2}}-\lambda_{a_{2}} \nabla \lambda_{a_{1}}\right) P_{i}^{0,0}\left(\lambda_{a_{2}}-\lambda_{a_{1}}\right), \quad 0 \leq i \leq r-1, \quad 1 \leq a \leq 6
$$

For a triangular face $f:$ Let $a_{1}, a_{2}$ and $a_{3}$ the vertices $f, f_{1}$ the other face containing edge $\left[a_{1}, a_{2}\right]$ and $f_{2}$ the other face containing $\left[a_{1}, a_{3}\right]$

$$
\begin{aligned}
& \left(\lambda_{a_{1}} \nabla \lambda_{a_{2}}-\lambda_{a_{2}} \nabla \lambda_{a_{1}}\right) \lambda_{f_{1}} P_{i j}(\hat{x}, \hat{y}, \hat{z}) \\
& \left(\lambda_{a_{1}} \nabla \lambda_{a_{3}}-\lambda_{a_{3}} \nabla \lambda_{a_{1}}\right) \lambda_{f_{2}} P_{i j}(\hat{x}, \hat{y}, \hat{z})
\end{aligned} \quad 0 \leq i+j \leq r-2
$$

with

$$
P_{i j}(\hat{x}, \hat{y}, \hat{z})=P_{i}^{0,0}\left(\lambda_{a_{2}}-\lambda_{a_{1}}\right) P_{j}^{0,0}\left(\lambda_{a_{3}}-\lambda_{a_{1}}\right)
$$

For interior functions :

$$
\begin{aligned}
& \left(\lambda_{1} \nabla \lambda_{4}-\lambda_{4} \nabla \lambda_{1}\right) \lambda_{2} \lambda_{3} P_{i j k}(\hat{x}, \hat{y}, \hat{z}) \\
& \left(\lambda_{1} \nabla \lambda_{2}-\lambda_{2} \nabla \lambda_{1}\right) \lambda_{3} \lambda_{4} P_{i j k}(\hat{x}, \hat{y}, \hat{z}) \quad 0 \leq i+j+k \leq r-3 \\
& \left(\lambda_{1} \nabla \lambda_{3}-\lambda_{3} \nabla \lambda_{1}\right) \lambda_{2} \lambda_{4} P_{i j k}(\hat{x}, \hat{y}, \hat{z})
\end{aligned}
$$

with

$$
\begin{aligned}
P_{i j k}(\hat{x}, \hat{y}, \hat{z})= & P_{i}^{0,0}\left(\frac{2 \hat{x}}{1-\hat{y}-\hat{z}}-1\right) P_{j}^{2 i+1,0}\left(\frac{2 \hat{y}}{1-\hat{z}}-1\right) \\
& (1-\hat{y}-\hat{z})^{i}(1-\hat{z})^{j} P_{k}^{2(i+j+1), 0}(2 \hat{z}-1)
\end{aligned}
$$

By construction, these hierarchical functions are a base of the optimal spaces $\hat{P}_{r}$ for each element, and the restriction on triangular faces and quadrilateral faces coincide if the orientation is the same. For quadrilateral faces, when the orientation is different, signs have to be considered. For triangular faces, a linear operator, involving degrees of freedom associated with the interior of the face, is considered.

Remark 4. By using the first family of Nédélec with variable order, it is possible to obtain the optimal family. This property is easy to observe since the hp basis functions for the optimal first family have the same form as the first family. Only the bounds over $i, j$ and $k$ are changed. For example, in the case of pyramids, hierarchicals functions of the first family space $\hat{P}_{r}^{1}$ are almost the same as the functions presented for $\hat{P}_{r}$, with these new bounds :

$$
0 \leq i \leq r, 0 \leq j \leq r-1
$$

set only for the functions associated with the quadrilateral base. 


\section{Comparison With Other Pyramidal Elements}

In this section, a comparison with previous elements proposed in the literature is performed.

- For $r=1$, the first family space $\hat{P}_{r}^{1}$ (see remark 1 ) is exactly the same as proposed in (Coulomb et al., 1997), (Graglia et al., 1999), (Doucet, 2008), (Nigam and Phillips, 2010a).

- The finite element space proposed in (Gradinaru and Hiptmair, 1999) is the same, if we change basis functions into the followings $\gamma_{6}$ and $\gamma_{7}$ :

$$
\gamma_{6}=\left[\begin{array}{c}
-z+\frac{y z}{1-z} \\
\frac{x z}{1-z} \\
x-\frac{x y}{1-z}+\frac{x y z}{(1-z)^{2}}
\end{array}\right], \gamma_{7}=\left[\begin{array}{c}
\frac{y z}{1-z} \\
-z+\frac{x z}{1-z} \\
y-\frac{x y}{1-z}+\frac{x y z}{(1-z)^{2}}
\end{array}\right]
$$

- In (Nigam and Phillips, 2010a), the vertical basis functions must be corrected to ensure that tangential restrictions are polynomial on triangular faces:

$$
\tilde{F}_{e_{1}}=\left\{\frac{1}{(1+z)^{k+1-\gamma}}\left[\begin{array}{c}
-z(y-1) \\
-z(x-1) \\
(x-1)(y-1)
\end{array}\right], 0 \leq \gamma \leq k-1\right\}
$$

Once this correction is applied, it is easy to check the space generated by basis functions of the first family $\hat{P}_{r}^{1}$ associated with edges and faces (by adapting basis functions of proposition 2) is the same space generated by edge and face basis functions proposed in (Nigam and Phillips, 2010a). Therefore the difference between our finite element space and Nigam \& Phillips' one is due to interior basis functions. The proposed interior space in (Nigam and Phillips, 2010a) contains $3 r(r-1)^{2}$ degrees of freedom, therefore $r(r-1)(4 r-5) / 2$ more degrees of freedom compared to $\hat{P}_{r}^{1}$, and the same convergence rate is observed.

- The second space proposed by (Nigam and Phillips, 2010b) is much smaller, since its dimension is equal to $r\left(2 r^{2}+7 r+7\right) / 2$, that is $r(r-1)$ less degrees of freedom than $\hat{P}_{r}^{1}$. However, this second space does not include the first-order optimal space $\hat{P}_{1}$. Indeed the following basis functions are not included:

$$
\left[\begin{array}{c}
\frac{\hat{y}^{2}}{1-\hat{z}} \\
0 \\
\frac{\hat{x} \hat{y}^{2}}{(1-\hat{z})^{2}}
\end{array}\right]\left[\begin{array}{c}
0 \\
\frac{\hat{x}^{2}}{1-\hat{z}} \\
\frac{\hat{x}^{2} \hat{y}}{(1-\hat{z})^{2}}
\end{array}\right]
$$

Since $\mathcal{R}_{r}$ is included, the space proposed in (Nigam and Phillips, 2010b) will provide an optimal convergence for affine pyramids, but will be not consistent for non-affine pyramids.

- Zaglmayr cited by (Demkowicz et al., 2007) propose a space presented as a Nédélec's second family space. However a practical expression of this space is difficult to obtain, and basis functions are not provided. Moreover, it seems that the dimension of space contains four degrees of freedom for $r=1$ and 21 degrees of freedom for $r=2$, which is problematic because 21 is less than the number of degrees of freedom needed to ensure the tangential continuity with the other types of faces. We hope that a future publication of Zaglmayr will clarify the situation.

- Second-order basis functions proposed by (Coulomb et al., 1997) do not seem to be appropriate, since basis functions related to faces vanish completely on other faces, whereas only the tangential trace should vanish. Furthermore, spurious modes are observed with these basis functions, this observation is also confirmed by (Marais and Davidson, 2008). 
- (Graglia et al., 1999) propose nodal basis functions on the pyramid. These functions are constructed from the first-order basis functions of $\hat{P}_{1}^{1}$ by multiplying them by polynomials, whereas it should involve rational fractions. As a result, the optimal space $\hat{P}_{1}$ is never included by these functions, leading to non-consistent space with non-affine pyramids. Moreover, spurious modes are observed with affine pyramids, as stated by (Marais and Davidson, 2008).

- (Marais and Davidson, 2008) propose to split pyramids in two tetrahedra. A similar approach (Bluck and Walker, 2008) has been proposed for continuous elements, but the extension to higher order is non consistent for non-affine pyramids (see (Bergot et al., 2010)). Therefore we did not test this approach for edge elements.

The table Tab. 1 summarizes the properties of the different spaces.

Table 1: Inclusion of Nigam/Phillips, Graglia and Zgainski finite element spaces for $r \geq 2$

\begin{tabular}{|c|c|c|}
\hline Space $V_{r}$ & Inclusion in first family $\hat{P}_{r}^{1}$ & Inclusion in optimal first family $\hat{P}_{r}$ \\
\hline Zgainski & $\hat{P}_{1}^{1} \subset V_{2}$ & $\hat{P}_{1} \subset V_{2} \subset \hat{P}_{3}$ \\
Graglia & $\hat{P}_{1}^{1} \subset V_{2}$ & $\hat{P}_{1} \subsetneq V_{2}$ \\
(Nigam and Phillips, 2010a) & $\hat{P}_{r}^{1} \subset V_{r}$ & $\hat{P}_{r-1} \supset V_{r}$ \\
(Nigam and Phillips, 2010b) & $\hat{P}_{1}^{1} \subset V_{1}$ & $\hat{P}_{1} \subsetneq V_{r}$ \\
\hline
\end{tabular}

A dispersion analysis (see (Bergot et al., 2010) for more details) has been performed for the different finite element spaces for a mesh made of a repeated cell composed of non-affine pyramids and affine pyramids, as shown in Fig. 7. The dispersion error obtained for these kinds of meshes is displayed in Fig. 8. For affines pyramids, Nigam \& Phillips' spaces and optimal spaces give a dispersion error in $O\left(h^{2 r}\right)$ as expected, whereas Graglia and Zgainski basis functions give an error in $O\left(h^{2}\right)$ only. For non-affine pyramids, optimal spaces give again a dispersion error in $O\left(h^{2 r}\right)$, whereas the first space of Nigam \& Phillips provide an error in $O\left(h^{2 r-2}\right)$, the second space being non-consistent.

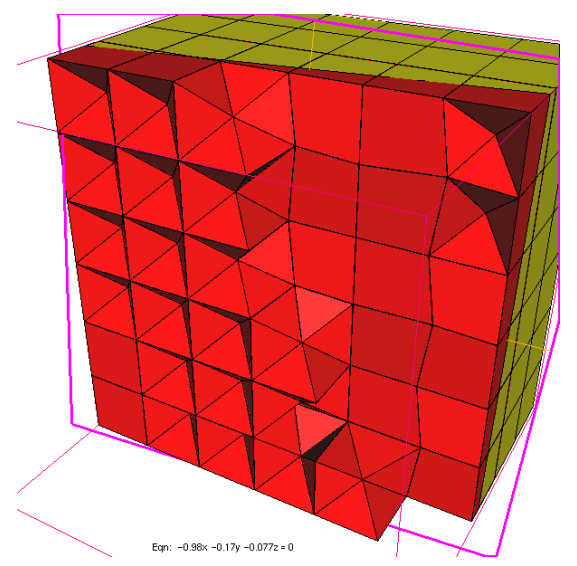

Figure 7: Pyramidal mesh used for the dispersion analysis.

We have also checked the accuracy of the source problem (1) for the same family of meshes, the error obtained between the numerical solution and a reference solution is displayed in Fig. 8. We can see that the optimal finite element space gives a better accuracy than the spaces described in (Coulomb et al., 1997), (Nigam and Phillips, 2010a), (Graglia et al., 1999). Due to the presence of spurious modes, the spaces of (Coulomb et al., 1997) and (Graglia et al., 1999) may lead to non-convergent solutions. We have numerically computed eigenmodes in a cubic cavity, we observed spurious modes for these spaces, whereas no spurious mode was observed for the optimal finite element spaces or spaces proposed by (Nigam and Phillips, 2010a). Numerical properties of different spaces are summarized in table Tab. 2. By compatibility, we mean the compatibility with other elements (hexahedra and tetrahedra). 

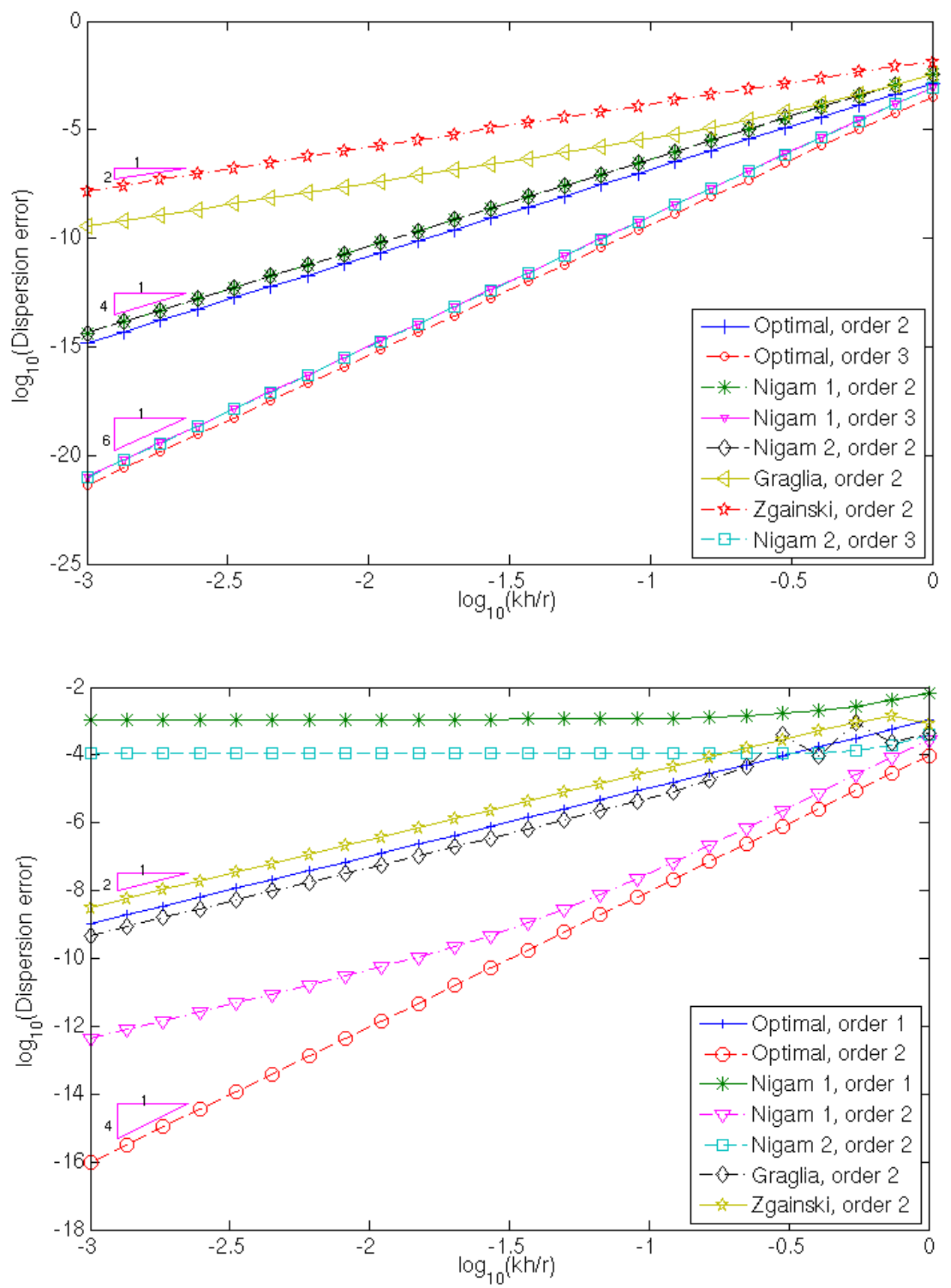

Figure 8: Dispersion error in log-log scale for a mesh comprising affine pyramids for the curve on top, and for a mesh comprising affines and non-affine pyramids for the bottom curve. 


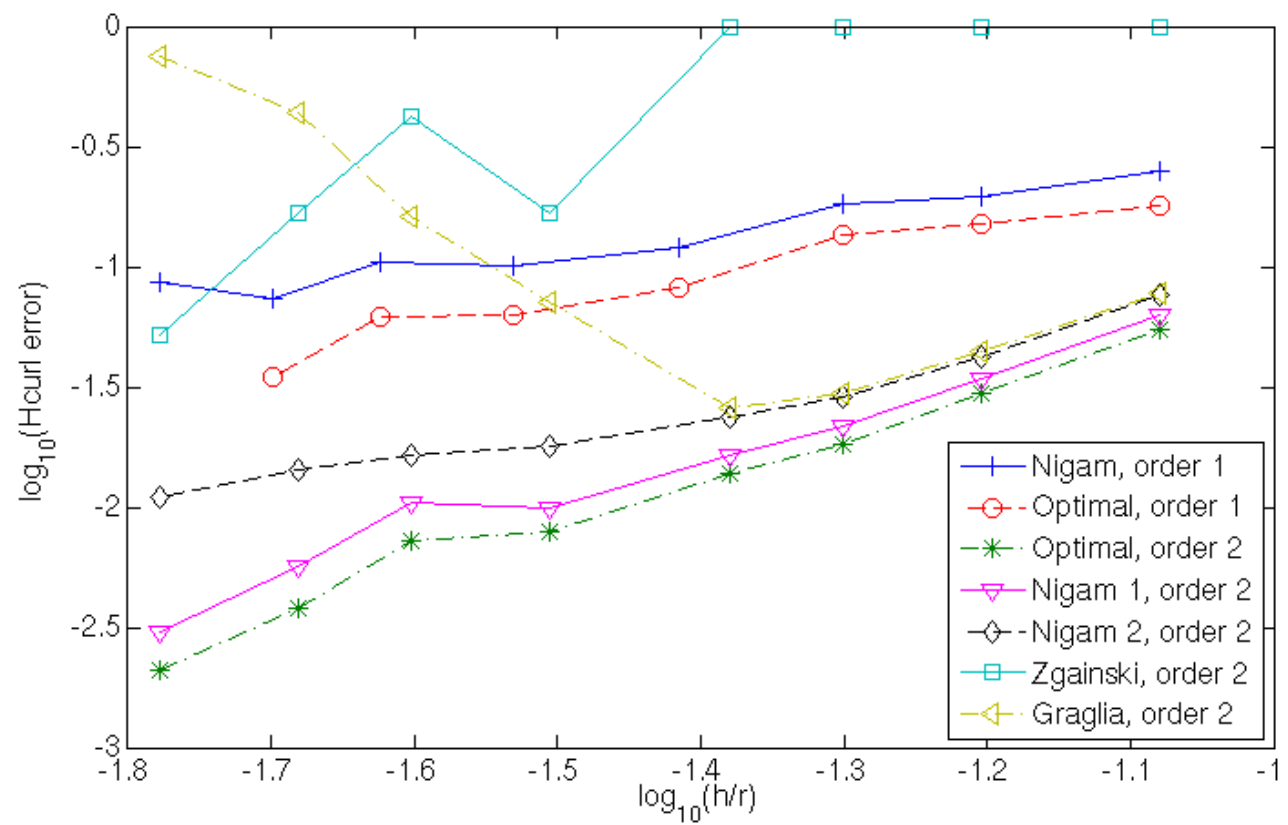

Figure 9: $H(\mathrm{curl})$-error in log-log scale for a mesh comprising affines and non-affine pyramids (Gaussian source inside a cubic cavity).

Table 2: Properties of different finite element spaces

\begin{tabular}{|c|c|c|c|c|c|}
\hline Property & Zgainski, $r=2$ & Graglia, $r=2$ & Nigam/Phillips 1 & Nigam/Phillips 2 & Optimal \\
\hline Convergence with affine pyramids & $O(h)$ & $O(h)$ & $O\left(h^{r}\right)$ & $O\left(h^{r}\right)$ & $O\left(h^{r}\right)$ \\
\hline Convergence with non-affine pyramids & $O(h)$ & $O(1)$ & $O\left(h^{r-1}\right)$ & $O(1)$ & $O\left(h^{r}\right)$ \\
\hline Spurious modes & yes & yes & no & no & no \\
\hline Compatibility & no & yes & yes & yes & yes \\
\hline
\end{tabular}




\section{Numerical Results}

In this section, the following scattering problem will be solved

$$
\left\{\begin{array}{l}
-\omega^{2} \varepsilon E+\operatorname{curl}\left(\frac{1}{\mu} \operatorname{curl} E\right)=f \text { in } \Omega \\
E \times n=-E^{\text {inc }} \times n \text { on } \Gamma \\
\operatorname{curl} E \times n=i k(n \times E) \times n \text { on } \Sigma
\end{array}\right.
$$

where $n$ is the normal to the boundary, $\Omega$ the computational domain, $\Gamma$ the scattered object and $\Sigma$ the external boundary.

The wave number $k$ is equal to

$$
k=\omega \sqrt{\varepsilon \mu}
$$

And the incident field is chosen as a plane wave

$$
E^{\text {inc }}=E^{0} \exp (i \vec{k} \cdot x)
$$

where $\vec{k}$ is the wave vector and $E_{0}$ the polarization (we will chose $E^{0}=e_{x}$ ). Here, an homogeneous media will be treated, i.e :

$$
\varepsilon=\mu=1
$$

The boundary condition set on $\Sigma$ is the Silver-Muller condition.

As a first validation, we consider the scattering of the sphere of radius 2 and $\omega=2 \pi$. A family of hybrid meshes (generated with Harpoon mesh generator) of the cube $[-3,3]$ containing a sphere of radius 2 (see Fig. 10). The

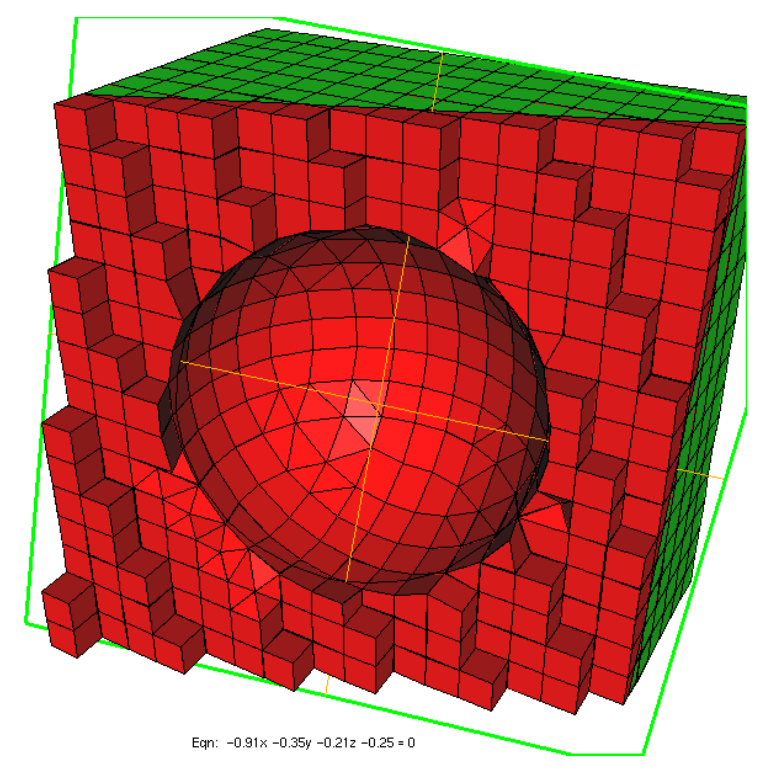

Figure 10: Example of hybrid mesh used for the scattering by a sphere

solution obtained is displayed in figure Fig. 11. On this simple shape, curved elements are used to approximate accurately the geometry. A reference solution is computed on a purely hexahedral mesh with $r=8$ and containing about 4 million degrees of freedom. The convergence of the numerical solution obtained with Nédélec first family space $\hat{P}_{r}^{1}$ and optimal finite element spaces is plotted in figure Fig. 12. It can be noticed that optimal finite element spaces provide a better accuracy for a same mesh size. However, it is important to remind that the number of degrees of freedom will be more important with the optimal spaces. 

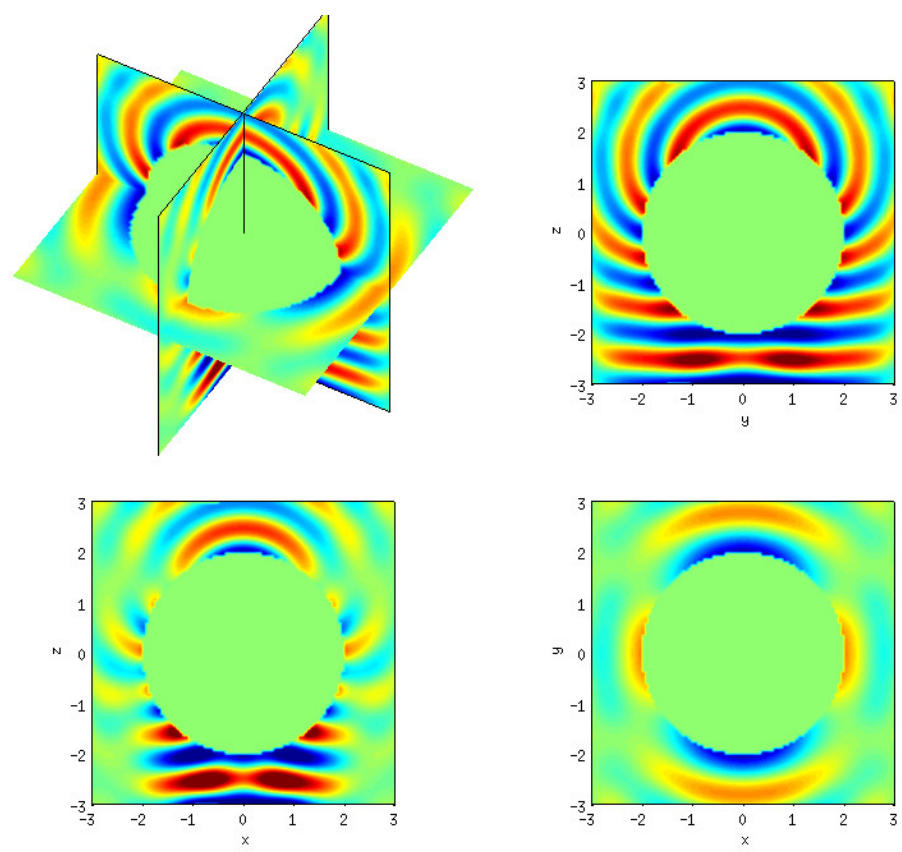

Figure 11: Real part of the diffracted field $E_{x}$ for a sphere of radius 2.

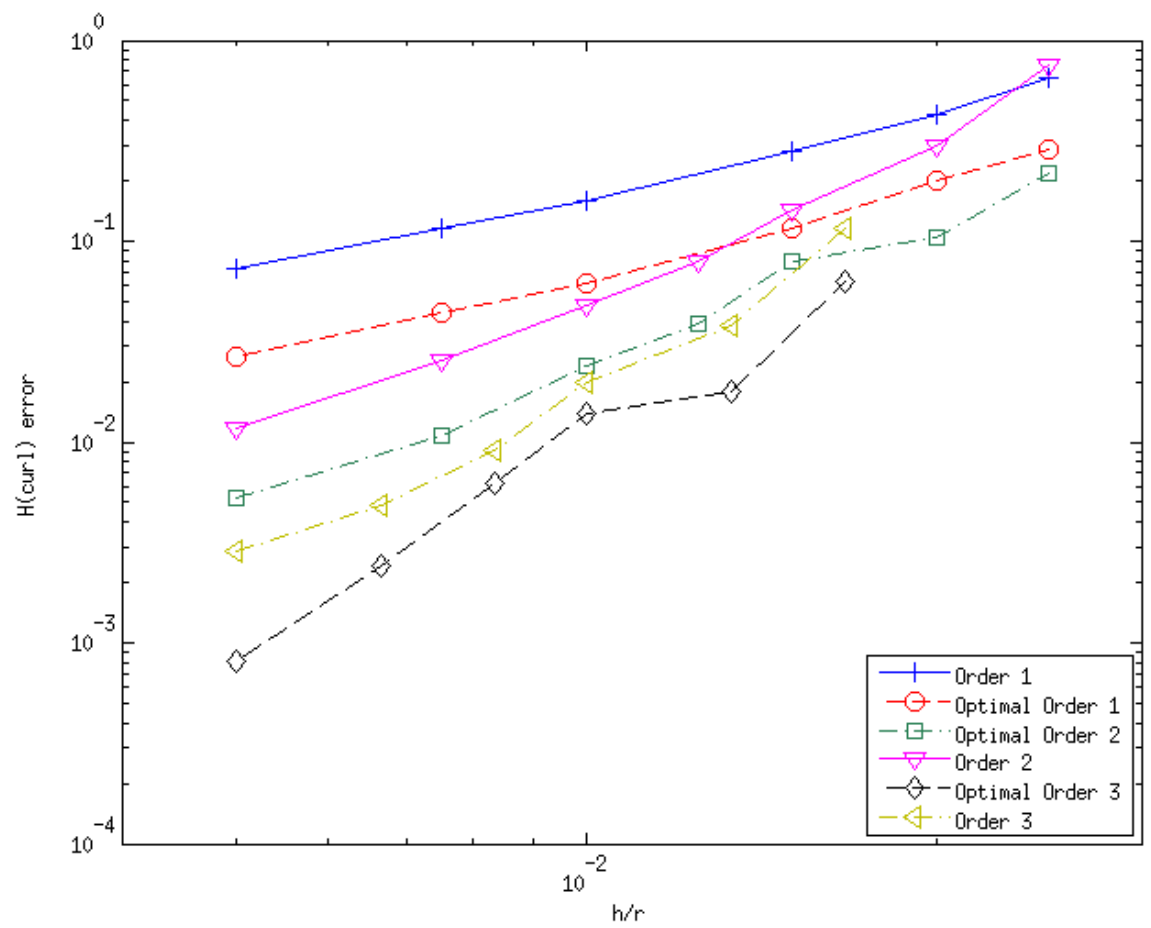

Figure 12: Convergence obtained for the perfectly conducting sphere with first family $\hat{P}_{r}^{1}$ and optimal first family $\hat{P}_{r}$ 
In order to validate hierarchical basis functions, we have used a variable order for the scattering of a satellite, the hybrid surface mesh of the object is displayed in figure Fig. 13. Because of thin solar panels and the presence of thin slots, some geometric details need very fine cells in order to be correctly approximated. In that regions, the geometric step is much smaller than the wavelength, and first or second-order approximation is sufficient, whereas an high order of approximation is used for large cells in order to fit the thumb rule " 10 points per wavelength". This rule is corrected since 10-th order element provide good results with 5 points per wavelength whereas first-order elements often need twenty or more points per wavelength. Thanks to that rule, an order of approximation is computed for each edge of the mesh, then the order associated with each face is set to the maximum among edges of the face. The order associated with the interior of the element is set to the maximum among edges of the element. In the hierarchical functions given in section 5.2, the order $r$ is actually different for each edge, face and interior of the element. The order associated with elements of the mesh is displayed in figure 14.

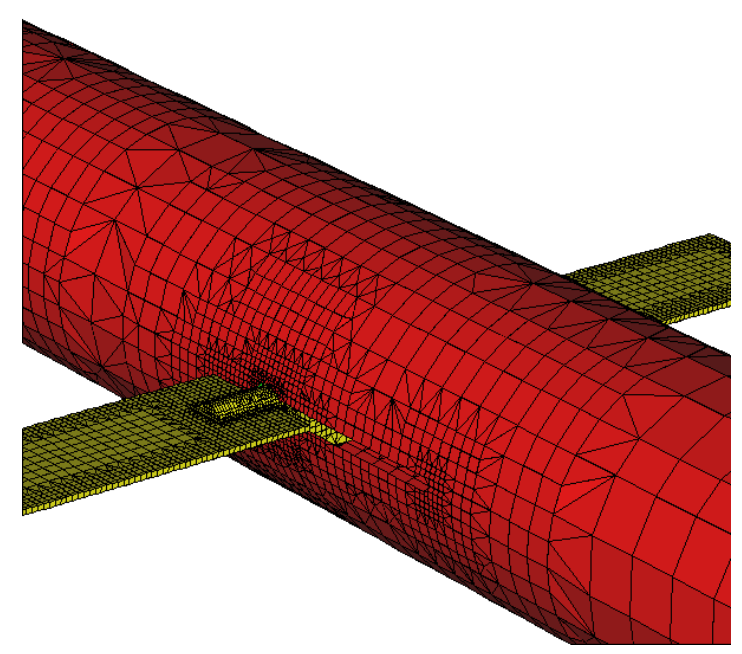

Figure 13: Surface of the hybrid mesh used for the scattering by a satellite

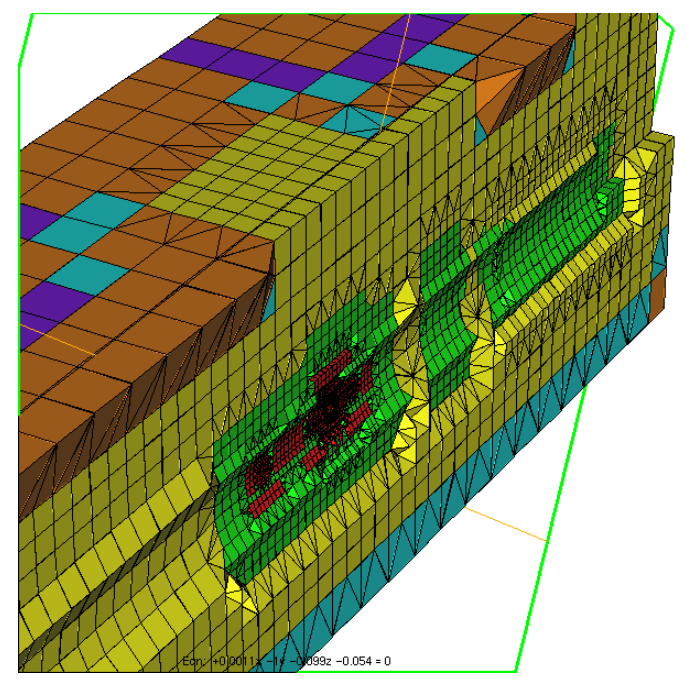

Figure 14: Hybrid mesh used for the scattering by a satellite. Each color is associated with a different order of approximation (red : 1, green : 2 , yellow : 3 , cyan : 4 , orange : 5 , purple : 6 ) 
The diffracted and total field are computed with Nédélec's first family $\hat{P}_{r}^{1}$ (see Fig 15).
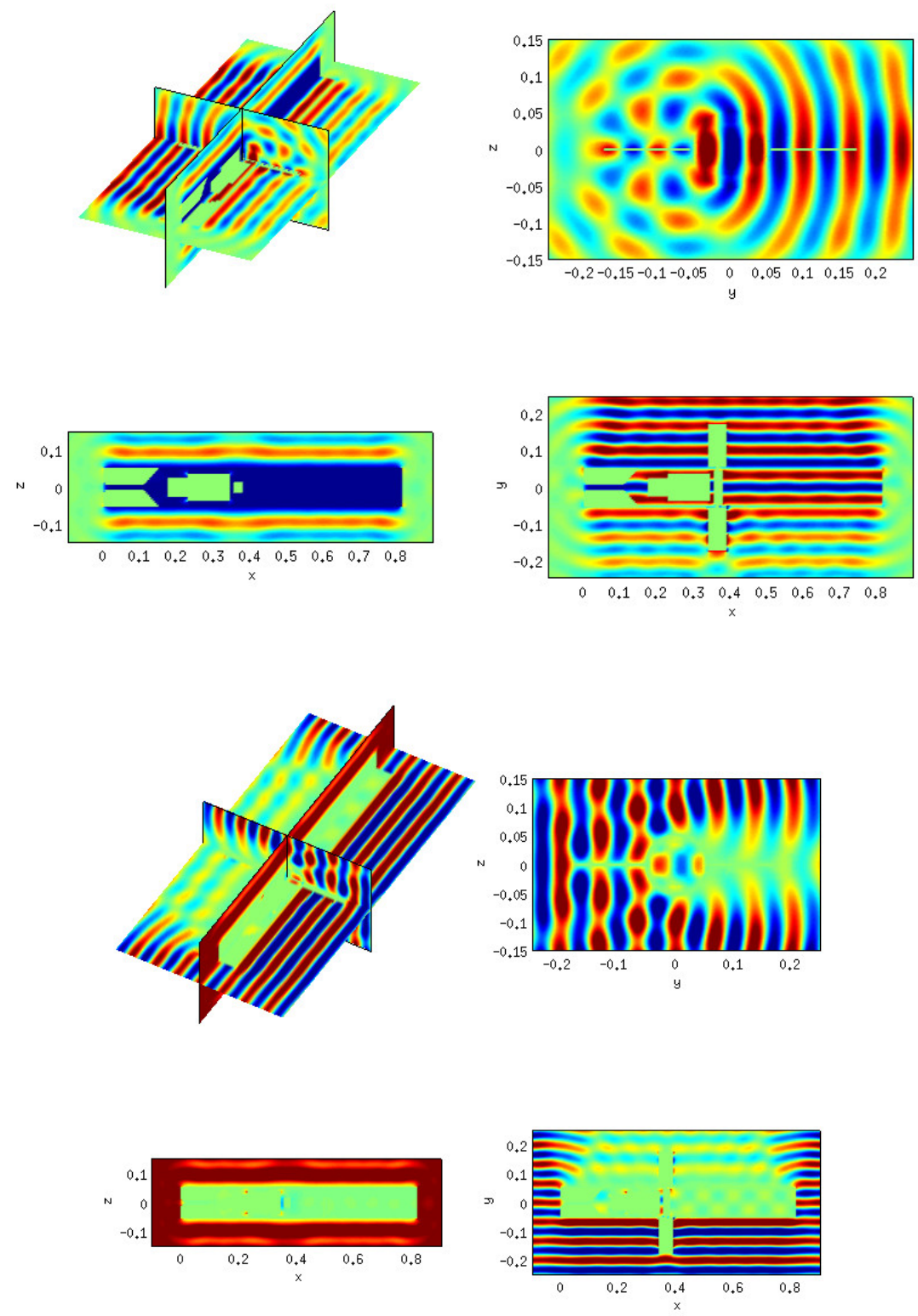

Figure 15: On top, real part of diffracted field $E_{x}$, and on bottom the total field. Scattering by a satellite

The mesh contains 35006 tetrahedra, 50390 hexahedra (40 659 affine hexahedra), 48865 pyramids (40 508 affine pyramids), 4582 wedges. With our strategy of adaptive order, the number of degrees of freedom is equal to 2570034. Because of the large amount of affine elements and the reduced number of degrees of freedom, the use of Nédélec's first family $\hat{P}_{r}^{1}$ seems relevant. 


\section{References}

Arnold, D. N., Boffi, D., Falk, R. S., 2005. Quadrilateral H(div) finite elements. SIAM J. Numer. Anal. 42 (6), 2429-2451.

Bedrosian, G., 1992. Shape functions and integration formulas for three-dimensional finite element analysis. Int. J. Numer. Meth. Eng. 35, 95-108.

Bergot, M., Cohen, G., Duruflé, M., 2010. Higher-order finite elements for hybrid meshes using new nodal pyramidal elements. J. Sci. Comput 42 (3), 345-381.

Beuchler, S., Pillwein, V., Schoberl, J., Zaglmayr, S., 2011. Sparsity optimized high order finite element functions on simplices. Numerical and Symbolic Scientific Computing: Progress and Prospects, 21-44.

Bluck, M., Walker, S., 2008. Polynomial basis functions on pyramidal elements. Com. Numer. Meth. Engn. 24, 1827-1837.

Cohen, G., Monk, P., 1998. Gauss point mass-lumping schemes for Maxwell's equations. NMPDE Journal 14 (1), 63-88.

Costabel, M., Dauge, M., 2002. Weighted regularization of Maxwell equations in polyhedral domains . A rehabilitation of nodal finite elements. Numer. Math. 93, 239-277.

Coulomb, J. C., Zgainski, F. X., Maréchal, Y., 1997. A pyramidal element to link hexahedral, prismatic and tetrahedral edge finite elements. IEEE Trans. Mag. 33 (2), 1362-1365.

Demkowicz, L., Kurtz, J., Pardo, D., Paszynski, M., Rachowicz, W., Zdunek, A., 2007. Computing With hp-Adaptive Finite Elements, Volume II. Chapman \& Hall/CRC.

Doucet, C., 2008. Approximation des champs électromagnétiques sur les maillages éléments finis hybrides conformes. Ph.D. thesis, Université Joseph Fourier.

Falk, R., Gatto, P., Monk, P., 2011. Hexahedral H(div) and H(curl) finite elements. ESAIM: M2AN 45 (1), 115-143.

Gradinaru, V., Hiptmair, R., 1999. Whitney elements on pyramids. Elec. Trans. Num. Anal. 8, 154-168.

Graglia, R. D., Wilton, D. R., Peterson, A. F., 1997. Higher order interpolatory vector bases for computational electromagnetics. IEEE Trans. Ant. Prop. 45 (3), 329-342.

Graglia, R. D., Wilton, D. R., Peterson, A. F., Gheorma, I.-L., 1999. Higher order interpolatory vector bases on pyramidal elements. IEEE Trans. Ant. Prop. 47 (5), 775-782.

Hesthaven, J., Teng, C., 2000. Stable spectral methods on tetrahedral elements. SIAM J. Numer. Anal. 21 (6), 2352-2380.

Houston, P., Perugia, I., Schneebeli, A., Schoetzau, D., 2005. Interior penalty method for the indefinite time-harmonic Maxwell equations. Numer. Math. 100, 485-518.

Marais, N., Davidson, D., 2008. Conforming arbitrary order hexahedral/tetrahedral hybrid discretisation. Electronics Letters 44 (24).

Monk, P., 2002. Finite element methods for Maxwell's equations. Oxford Science Publication.

Nédélec, J. C., 1980. Mixed finite elements in $\mathbb{R}^{3}$. Numer. Math. 35 (3), 315-341.

Nigam, N., Phillips, J., 2010a. High-order finite elements on pyramids: approximation spaces, unisolvency and exactness. accepted in IMA J. of Nu. Anal.

Nigam, N., Phillips, J., 2010b. Numerical integration for high order pyramidal finite elements. in revision.

Šolín, P., Segeth, K., Dolezel, I., 2003. Higher-Order Finite Elements Methods. Chapman \& Hall/CRC.

Xin, J., Guo, N., Cai, W., 2011. On the construction of well-conditioned hierarchical bases for tetrahedral h(curl)-conforming nédélec element. J. Comp. Math. 29, 526-542.

Zaglmayr, S., 2006. High order finite elements for electromagnetic field computation. Ph.D. thesis, Johannes Kepler University, Linz Austria. 